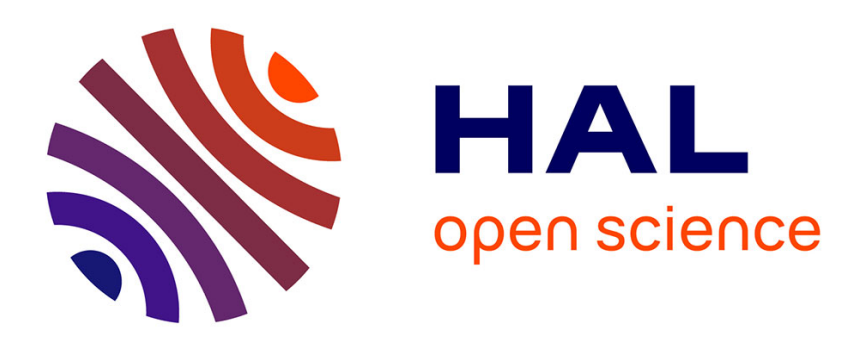

\title{
Algébrisation des variétés analytiques complexes et catégories dérivées.
}

Bertrand Toen, Michel Vaquié

\section{To cite this version:}

Bertrand Toen, Michel Vaquié. Algébrisation des variétés analytiques complexes et catégories dérivées.. Mathematische Annalen, 2008, 342 (4), pp.789-831. 10.1007/s00208-008-0257-9 . hal00772940

\section{HAL Id: hal-00772940 \\ https://hal.science/hal-00772940}

Submitted on 11 Jan 2013

HAL is a multi-disciplinary open access archive for the deposit and dissemination of scientific research documents, whether they are published or not. The documents may come from teaching and research institutions in France or abroad, or from public or private research centers.
L'archive ouverte pluridisciplinaire HAL, est destinée au dépôt et à la diffusion de documents scientifiques de niveau recherche, publiés ou non, émanant des établissements d'enseignement et de recherche français ou étrangers, des laboratoires publics ou privés. 


\title{
Algébrisation des variétés analytiques complexes et catégories dérivées
}

\author{
Bertrand Toën et Michel Vaquié \\ Institut de Mathématiques de Toulouse \\ UMR CNRS 5219 \\ Université Paul Sabatier, Bat 1R2 Toulouse Cedex 9
}

Mars 2007

\section{Résumé}

Soit $X$ un espace analytique complexe compact et lisse. Nous démontrons que $X$ est algébrisable si et seulement si sa dg-catégorie dérivée cohérente bornée est saturée.

\section{Table des matières}

1 Introduction

2 Rappels sur le foncteur d'analytification 3

2.1 Changements de sites pour les faisceaux . . . . . . . . . . . . . . . . 3

2.2 Contextes et champs géométriques . . . . . . . . . . . . . . . . . 6

2.3 Analytification des espaces algébriques . . . . . . . . . . . . . . . . 11

3 Catégories dérivées des espaces analytiques 14

4 Analytification des espaces de modules d'objets dans des dg-catégories 18

4.1 Rappels sur les espaces de modules de dg-modules simples . . . . . . . . . . . . . 18

4.2 Description de l'espace $M(B)^{a n}$. . . . . . . . . . . . . . . . . . . . . . 22

5 Le théorème de caractérisation $\quad 27$

5.1 Algébrisation de $M(X)$. . . . . . . . . . . . . . . . . . . . . . . . 27

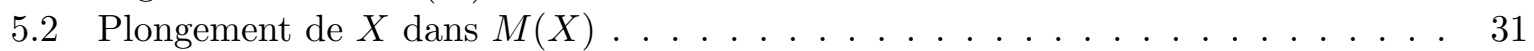

5.3 Algébrisation de $X \ldots \ldots \ldots$. . . . . . . . . . . . . . . . . 33

A Dg-catégories saturées et catégories triangulées saturées 34

B Catégories dérivées des espaces algébriques propres et lisses 35 


\section{Introduction}

Pour un schéma $X$ propre et lisse sur $\operatorname{Spec} \mathbb{C}$ (et plus généralement pour un espace algébrique complexe propre et lisse $X$ ), la catégorie triangulée $D_{\text {parf }}(X)$ des complexes parfaits de $\mathcal{O}_{X^{-}}$ modules possède de remarquables propriétés de finitudes. Dans $[\mathrm{B}-\mathrm{V}]$ il est démontré que $D_{\text {parf }}(X)$ est saturé, au sens ou ses Ext globaux sont de dimension finie et de plus tout foncteur cohomologique de type fini $H: D_{\text {parf }}(X)^{o p} \longrightarrow V e c t$ est représentable. De plus, Bondal et Van den Bergh démontrent aussi que si $X$ est une surface complexe compacte qui ne possède pas des courbes compactes alors $D_{\text {parf }}(X)$ n'est pas saturée. Ils suggèrent alors que toute variété complexe compacte $X$ dont la catégorie dérivée parfaite est saturée est algébrisable (voir $[\mathrm{B}-\mathrm{V}$, Rem . 5.6.2]). Le but de ce travail est de démontrer cette assertion lorsque l'on remplace la cadre étriqué des catégories triangulées par celui plus flexible des dg-catégories.

Pour $X$ une variété complexe compacte, la catégorie triangulée $D_{\text {parf }}(X)$ est la catégorie homotopique d'une dg-catégorie naturelle $L_{p a r f}(X)$ (voir [To-Va] et la définition 3.7). De plus, une notion de $d g$-catégorie saturée est introduite dans [To-Va], qui est proche de celle de catégorie triangulée saturée (voir l'appendice A pour une comparaison). Le théorème principal de ce travail est le suivant.

Théorème 1.1 Soit $X$ un espace analytique compact et lisse (i.e. une variété complexe compacte et lisse). Alors $X$ est algébrisable (par un espace algébrique) si et seulement si la dgcatégorie $L_{\text {parf }}(X)$ est saturée.

La nécessité de la condition est bien connue, nous rappellerons une esquisse de preuve dans l'appendice B. La partie difficile est la suffisance, dont la preuve est une application du résultat principal de [To-Va] qui affirme le caractère algébrique du champ des modules des objets dans une dg-catégorie saturée. Plus précisèment, nous commencerons par considérer l'espace algébrique $M$ des objets simples dans la dg-catégorie $L_{\text {parf }}(X)$. L'existence de cet espace algébrique est assurée par les résultats de [To-Va] (voir le théorème 4.8). Nous montrerons ensuite que l'analytifié $M^{a n}$ de cet espace algébrique est un espace de modules (au sens analytique) pour les complexes parfaits et simples sur $X$ (voir Prop. 5.4). Nous considérons alors le morphisme $j: X \longrightarrow M^{a n}$ qui à un poit $x$ de $X$ associe le faisceau gratte-ciel $k(x)$, et nous montrons que $j$ est une immersion ouverte (voir Prop. 5.6). Enfin, en retreignant les fonctions méromorphes de $M^{a n}$ à $X$ nous déduisons que $X$ est un espace de Moishezon et donc est un espace algébrique (voir Prop. $5.7)$.

Ce travail est découpé en quatres sections et trois appendices. Dans une première partie nous rappelons quelques faits sur les foncteurs de changement de sites pour les faisceaux. Nous introduirons aussi la notion de contextes géométriques et de faisceaux géométriques, qui nous permettront de construire le foncteur d'analytification. La section suivante rappelle quelques résultats de la théorie des catégories dérivées en géométrie analytique. Ces deux premières sections consistent essentiellement en des rappels de résultats connus, et le lecteur pourra commencer sa lecture à la section $\S 4$ et consulter les sections $\S 2,3$ lorsque cela est nécessaire. La 
section $\S 4$ présente d'une part le résultat d'existence d'un espace algébrique de modules pour les dg-modules simples sur une dg-algèbre propre et lisse, ainsi qu'une description de l'analytifié de cet espace. Enfin, la section $\S 5$ présente la preuve du théorème principal.

Dans les appendices, le lecteur trouvera tout d'abord une comparaison entre les notions de catégories triangulées saturées et de dg-catégories saturées, ce qui permet de remettre notre théorème dans le cadre de $[\mathrm{B}-\mathrm{V}]$. L'appendice $\mathrm{B}$ présente très brièvement une preuve de la nécessité du théorème 1.1, qui est probablement un fait déjà connu. Enfin, l'appendice $\mathrm{C}$ présente un formalisme qui permet de manipuler des catégories dérivées non bornées à l'aide de techniques de catégories de modèles. L'existence de cette structure de modèles sera utilisée tout au long de ce travail.

\section{Rappels sur le foncteur d'analytification}

Dans cette section nous rappellerons les constructions de foncteur de changements de sites pour les faisceaux. Nous introduirons aussi la notion de contextes géométriques et de faisceaux géométriques relatifs à ces contextes. Nous montrerons alors que sous certaines conditions les foncteurs de changement de sites préservent les objets géométriques. Nous appliquerons cela aux contextes algébrique et analytique, et nous obtiendrons ainsi un foncteur d'analytification des espaces algébriques vers les espaces analytiques. Mises à part les notions de contextes et de faisceaux géométriques les résultats de cette section sont bien connus.

\subsection{Changements de sites pour les faisceaux}

Soit $C$ une catégorie possédant des limites finies. Nous notons $\operatorname{Pr}(C)$ la catégorie des préfaisceaux sur $C$, c'est-à-dire la catégorie des foncteurs de $C^{o p}$ à valeurs dans la catégorie Ens des ensembles.

Pour tout $x \in C$ nous définissons le préfaisceau $h_{x}$ par

$$
h_{x}(y)=C(y, x) .
$$

Le foncteur $h: C \longrightarrow \operatorname{Pr}(C)$ est un pleinement fidèle et nous appelons préfaisceau représentable tout préfaisceau dans son image essentielle. Tout préfaisceau $F \in \operatorname{Pr}(C)$ admet une résolution par des préfaisceaux représentables, c'est-à-dire peut être obtenu comme colimite de préfaisceaux représentables.

Un foncteur $f: C \rightarrow D$ définit une adjonction entre les catégories de préfaisceaux

$$
\operatorname{Pr}(C) \underset{f^{*}}{\stackrel{f !}{\gtrless}} \operatorname{Pr}(D)
$$

où le foncteur adjoint à droite $f^{*}$ est défini par $f^{*}(F)(x):=F(f(x))$ pour tout préfaisceau $F$ dans $S \operatorname{Pr}(D)$ et pour tout $x$ dans $C$. Le foncteur adjoint à gauche $f$ ! est défini sur les préfaisceaux représentables par $f_{!}\left(h_{x}\right):=h_{f(x)}$ pour tout $x$ dans $C$, et est étendu au préfaisceaux par extension de Kan à gauche.

Nous rappelons qu'un foncteur $f: C \longrightarrow D$ est dit exact à gauche s'il préserve les limites finies, c'est-à-dire s'il préserve les produits fibrés et l'objet final. 
Proposition 2.1 Soient $C$ et $D$ deux catégories possédant des limites finies, et $f: C \longrightarrow D$ un foncteur exact à gauche. Alors le foncteur induit $f_{!}: \operatorname{Pr}(C) \longrightarrow \operatorname{Pr}(D)$ est exact à gauche.

Preuve: Comme $C$ possède un objet final $*$ et que $f(*) \simeq *$, nous avons $*=h_{*}$ et $f_{!}(*) \simeq$ $h_{f(*)} \simeq h_{*}$.

Il nous reste à montrer que pour tout diagramme de préfaisceaux $F \longrightarrow H<G$, le morphisme naturel

$$
f_{!}\left(F \times_{H} G\right) \longrightarrow f_{!}(F) \times_{f_{!}(H)} f_{!}(G)
$$

est un isomorphisme dans $\operatorname{Pr}(D)$. Comme les colimites d'ensembles sont universelles (i.e. stables par changement de bases) elles sont aussi universelles dans la catégorie des préfaisceaux. De plus, comme tout préfaisceau peut être obtenu comme colimite de préfaisceaux représentables nous pouvons supposer que les préfaisceaux $F$ et $G$ sont de la forme respectivement $h_{x}$ et $h_{y}$. Nous sommes donc ramenés à montrer que le morphisme naturel

$$
f_{!}\left(h_{x} \times_{H} h_{y}\right) \longrightarrow h_{f(x)} \times_{f_{!}(H)}^{h} h_{f(y)}
$$

est un isomorphisme.

Nous rappelons que si $x, y$ et $z$ sont des objets de $C$ nous avons des isomorphismes naturels

$$
h_{x \times{ }_{z} y} \simeq h_{x} \times_{h_{z}} h_{y} .
$$

Par conséquent, comme le foncteur $f$ commute aux produits fibrés, nous trouvons le résultat dans le cas où $H$ est lui aussi représentable:

$$
f_{!}\left(h_{x} \times_{h_{z}} h_{y}\right) \simeq h_{f\left(x \times_{z} y\right)}=h_{f(x) \times_{f(z)} f(y)} \simeq f_{!}\left(h_{x}\right) \times_{f_{!}\left(h_{z}\right)} f_{!}\left(h_{y}\right) .
$$

De ceci, est du fait que les sommes sont disjointes dans $\operatorname{Pr}(C)$, il est facile de montrer le résultat pour le cas où $H$ est isomorphe dans $\operatorname{Pr}(C)$ à une somme de préfaisceaux représentables.

Venons en au cas général. Choisissons un épimorphisme $p: X_{0} \longrightarrow H$, avec $X_{0}$ isomorphe dans $\operatorname{Pr}(C)$ à une somme de représentables. Soit

$$
X_{1}:=X_{0} \times_{H} X_{0} \rightrightarrows X_{0}
$$

la relation d'équivalence sur $X_{0}$ définie par $p$. Comme $p$ est un épimorphisme dans $\operatorname{Pr}(C)$ le morphisme naturel

$$
X_{0} / X_{1} \longrightarrow H,
$$

du quotient de $X_{0}$ par la relation $X_{1}$ dans $H$, est un isomorphisme dans $\operatorname{Pr}(C)$ (cela se voit par exemple en considérant les fibres de $X_{0} / X_{1} \longrightarrow H$, qui sont des co-égaliseurs des deux projections $Z \times Z \rightrightarrows Z$ pour un certain ensemble $Z$ non vide).

Soit $Y_{i}:=f_{!}\left(X_{i}\right)$ pour $i=0,1$, et considérons les morphismes naturels

$$
Y_{1} \rightrightarrows Y_{0} .
$$

Nous déduisons de la proposition 2.1 dans le cas où $H$ est une somme de représentables que $Y_{1}$ est encore une relation d'équivalence sur $Y_{0}$. De plus, comme $f_{\text {! }}$ est adjoint à gauche il commute aux colimites, et on a

$$
Y_{0} / Y_{1} \simeq f_{!}\left(X_{0} / X_{1}\right) \simeq f_{!}(H) .
$$


Par conséquent nous avons

$$
f_{!}\left(X_{0} \times_{H} X_{0}\right) \simeq f_{!}\left(X_{1}\right)=Y_{1} \simeq Y_{0} \times_{f_{!}(H)} Y_{0} \simeq f_{!}\left(X_{0}\right) \times_{f_{!}(H)} f_{!}\left(X_{0}\right) .
$$

Comme le morphisme $p: X_{0} \rightarrow H$ est un épimorphisme, les morphismes $h_{x} \rightarrow H$ et $h_{y} \rightarrow H$ se factorisent par $X_{0}$. Par conséquent nous avons

$$
h_{x} \times_{H} h_{y} \simeq h_{x} \times_{X_{0}}\left(X_{0} \times_{H} X_{0}\right) \times_{X_{0}} h_{y} .
$$

D'après ce qui précède cela implique que le morphisme naturel

$$
f_{!}\left(h_{x} \times_{H} h_{y}\right) \longrightarrow f_{!}\left(h_{x}\right) \times_{f_{!}(H)} f_{!}\left(h_{y}\right)
$$

est un isomorphisme dans $\operatorname{Pr}(D)$.

Nous supposons maintenant que la catégorie $C$ est munie d'une topologie de Grothendieck $\tau$. La sous-catégorie de $\operatorname{Pr}(C)$ formée des faisceaux pour cette topologie sera notée $S h(C)$, et le foncteur de faisceautisation sera noté

$$
a: \operatorname{Pr}(C) \longrightarrow S h(C) .
$$

On rappelle que le foncteur $a$ est exact à gauche.

Proposition 2.2 Soient $C$ et $D$ deux catégories possédant des limites finies, et $\tau$ (resp. $\rho$ ) une topologie sur $C$ (resp. sur $D$ ). Soit $f: C \longrightarrow D$ un foncteur vérifiant les conditions suivantes.

1. Pour toute famille couvrant $\left\{U_{i} \longrightarrow X\right\}$ dans $C$, la famille $\left\{f\left(U_{i}\right) \longrightarrow f(X)\right\}$ est couvrante dans $D$.

2. Le foncteur $f$ est exact à gauche.

Alors le foncteur

$$
f^{*}: \operatorname{Pr}(D) \longrightarrow \operatorname{Pr}(C)
$$

préserve les faisceaux. De plus, le foncteur induit

$$
f^{*}: S h(D) \longrightarrow S h(C)
$$

possède un adjoint à gauche

$$
f_{!}^{a}:=a \circ f_{!}: \operatorname{Sh}(C) \stackrel{f_{!}}{\longrightarrow} \operatorname{Pr}(D) \stackrel{a}{\longrightarrow} \operatorname{Sh}(D)
$$

qui est exact à gauche.

Preuve: Le fait que $f_{!}^{a}$ défini comme le foncteur composé $a \circ f$ ! soit exact à gauche découle du fait que $a$ est exact à gauche et de la proposition 2.1. De plus, si l'on sait que $f^{*}$ préserve les faisceaux il est formel de voir que $f_{!}^{a}$ est adjoint à gauche de $f^{*}$. Il nous suffit donc de montrer que $f^{*}$ préserve les faisceaux. 
Pour cela, soit $F \in S h(D), X \in C$ et $\left\{U_{i} \longrightarrow X\right\}$ une famille couvrante. On considère le morphisme de préfaisceaux

$$
U:=\coprod_{i} h_{U_{i}} \longrightarrow h_{X}
$$

et $R:=U \times{ }_{h_{X}} U \rightrightarrows U$ la relation d'équivalence définie sur $U$. Dire que $f^{*}(F)$ possède la propriété de descente par rapport au recouvrement $\left\{U_{i} \longrightarrow X\right\}$ est équivalent à dire que le morphisme naturel

$$
\operatorname{Hom}_{\operatorname{Pr}(C)}\left(U / R, f^{*}(F)\right) \longrightarrow \operatorname{Hom}_{P r(C)}\left(h_{X}, f^{*}(F)\right)
$$

est bijectif. Or, par adjonction ce morphisme est isomorphe au morphisme naturel

$$
\operatorname{Hom}_{\operatorname{Pr}(D)}\left(f_{!}(U / R), F\right) \longrightarrow \operatorname{Hom}_{P r(D)}\left(f_{!}\left(h_{X}\right), F\right) .
$$

Par hypothèse sur $f$, le quotient $f_{!}(U / R)$ est isomorphe au quotient $V / R^{\prime}$, où $V:=\coprod_{i} h_{f\left(U_{i}\right)}$, et

$$
R^{\prime}:=V \times_{h_{f(X)}} V \rightrightarrows V
$$

est la relation d'équivalence définie par la projection $V \longrightarrow h_{f(X)}$. Ainsi, dire que le morphisme

$$
\operatorname{Hom}_{\operatorname{Pr}(D)}\left(f_{!}(U / R), F\right) \longrightarrow \operatorname{Hom}_{\operatorname{Pr}(D)}\left(f_{!}\left(h_{X}\right), F\right)
$$

est bijectif est équivalent à dire que le préfaisceau $F$ possède la condition de descente pour la famille couvrante $\left\{f\left(U_{i}\right) \longrightarrow f(X)\right\}$. Comme $F$ est un faisceau, cette condition de descente est satisfaite, et donc $f^{*}(F)$ possède la condition de descente pour $\left\{U_{i} \longrightarrow X\right\}$. Cela finit de montrer que $f^{*}(F)$ est un faisceau.

\subsection{Contextes et champs géométriques}

Définition 2.3 Un contexte géométrique est un triplet $(C, \tau, \mathbb{P})$, où $C$ est une catégorie, $\tau$ est une topologie de Grothendieck sur $C$ et $\mathbb{P}$ est une classe de morphismes dans $C$, vérifiant les conditions suivantes.

1. La topologie $\tau$ est sous-canonique (nous identifierons alors $C$ à une sous-catégorie pleine de la catégorie $S h(C)$ des faisceaux sur $C$ ).

2. La catégorie $C$ possède des limites finies et des sommes finies. De plus, les sommes sont disjointes dans $C$.

3. La classe de morphismes $\mathbb{P}$ est stable par composition, changements de bases, et contient les isomorphismes.

4. Pour toute famille couvrante $\left\{X_{i} \longrightarrow X\right\}$ dans $C$, il existe des morphismes $Y_{i} \longrightarrow X$ dans $\mathbb{P}$ et des diagrammes commutatifs

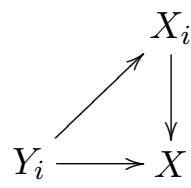


tels que nous ayons un épimorphisme de faisceaux

$$
\coprod_{i} h_{Y_{i}} \longrightarrow h_{X}
$$

5. Soit $f: Y \longrightarrow X$ un morphisme dans $C$ tel qu'il existe une famille couvrante $\left\{Y_{i} \longrightarrow Y\right\}$ dans $C$, telle que tous les morphismes $Y_{i} \longrightarrow Y$ et $Y_{i} \longrightarrow X$ soient dans $\mathbb{P}$. Alors le morphisme $f$ est dans $\mathbb{P}$.

6. Pour toute paire d'objets $X$ et $Y$ dans $C$ le morphisme naturel

$$
X \longrightarrow X \coprod Y
$$

est dans $\mathbb{P}$. De plus, la famille de morphismes

$$
\{X \longrightarrow X \coprod Y, Y \longrightarrow X \coprod Y\}
$$

est couvrante.

7. Soit $h: C \longrightarrow S h(C)$, le plongement de Yoneda de $C$ dans la catégorie des faisceaux sur $C$ pour la topologie $\tau$. Soit $X$ un objet de $C$ tel qu'il existe un isomorphisme dans $\operatorname{Sh}(C)$

$$
h_{X} \simeq F_{1} \coprod F_{2} .
$$

Alors il existe des objets $X_{1}$ et $X_{2}$ dans $C$ tels

$$
F_{1} \simeq h_{X_{1}} \quad F_{2} \simeq h_{X_{2}} .
$$

Fixons un contexte géométrique $(C, \tau, \mathbb{P})$, et considérons la catégorie $S h(C)$ des faisceaux sur le site $(C, \tau)$. Nous allons définir une sous-catégorie pleine de $S h(C)$ formée des espaces géométriques obtenus par recollement d'objets de $C$. La définition se fait par récurrence et suit les mêmes étapes que celle de [HAGII]. Pour cela rappelons que l'on dispose d'un plongement de Yoneda

$$
h: C \longrightarrow \operatorname{Pr}(C)
$$

qui se factorise en un foncteur pleinement fidèle (car $\tau$ est supposée sous-canonique)

$$
h: C \longrightarrow S h(C) .
$$

Nous identifierons la catégorie $C$ à son image essentielle par le foncteur $h$, et omettrons ainsi de mentioner $h$.

1. Nous commençons par décréter qu'un faisceau $F \in S h(C)$ est (-1)-géométrique s'il est représentable (i.e. isomorphe dans $S h(C)$ à un objet de la forme $h_{X}$ pour $X \in C$ ).

2. Un morphisme $f: F \longrightarrow G$ de faisceaux est (-1)-représentable si pour tout $X \in C$, et tout morphisme $X \longrightarrow G$ le faisceau $F \times_{G} X$ est (-1)-géométrique. 
3. Un morphisme (-1)-représentable $f: F \longrightarrow G$ est dans $\mathbb{P}$ si pour tout $X \in C$ et tout morphisme $X \longrightarrow G$ le morphisme induit entre faisceaux représentables

$$
F \times{ }_{G} X \longrightarrow X
$$

est dans $\mathbb{P}$.

4. Soit $n \geq 0$ et supposons que les trois notions de faisceaux $m$-géométriques, de morphismes $m$-représentables et de morphismes $m$-représentables et dans $\mathbb{P}$ soient définies pour tout $m<n$.

(a) Un faisceau $F$ est n-géométrique s'il existe une famille d'objets $\left\{U_{i}\right\}$ dans $C$ et un morphisme

$$
U:=\coprod_{i} U_{i} \longrightarrow F
$$

satisfaisant les deux conditions suivantes

i. Le morphisme $U \longrightarrow F$ est un épimorphisme de faisceaux.

ii. Chacun des morphismes $U_{i} \longrightarrow F$ est $(n-1)$-représentable et dans $\mathbb{P}$.

La donnée des $U_{i}$ et du morphisme

$$
\coprod_{i} U_{i} \longrightarrow F
$$

sera appelée un n-atlas pour $F$.

(b) Un morphisme $f: F \longrightarrow G$ est n-représentable si pour tout $X \in C$ et tout morphisme $X \longrightarrow G$ le faisceau $F \times_{G} X$ est $n$-géométrique.

(c) Un morphisme $n$-représentable $f: F \longrightarrow G$ est dans $\mathbb{P}$ si pour tout $X \in C$ et tout morphisme $X \longrightarrow G$ il existe un $n$-atlas $\left\{U_{i}\right\}$ de $F \times{ }_{G} X$ tel que tous les morphismes induits

$$
U_{i} \longrightarrow X
$$

soient dans $\mathbb{P}$.

Nous posons alors la définition suivante.

Définition 2.4 1. Un faisceau $F \in S h(C)$ est géométrique s'il est n-géométrique pour un certain $n$.

2. Un morphisme de faisceaux $f: F \longrightarrow G$ est dans $\mathbb{P}$ s'il est $n$-représentable et dans $\mathbb{P}$ pour un certain $n$.

On remarque que le procédé inductif de la définition précédente s'arrète en réalité à $n=1$.

Lemme 2.5 Si $n>1$, alors tout faisceau n-géométrique est $(n-1)$-géométrique. 
Preuve: Soit $F$ un faisceau $n$-géométrique et

$$
U:=\coprod_{i} U_{i} \longrightarrow F
$$

un $n$-atlas. Nous allons montrer que les morphismes $U_{i} \longrightarrow F$ sont en fait 0-géométrique. Pour cela, soit $X \in C$ et $X \longrightarrow F$ un morphisme. Le faisceau $U_{i} \times_{F} X$ est un sous-faisceau de $U_{i} \times X$. Soit

$$
\coprod_{j} V_{i, j} \longrightarrow U_{i} \times_{F} X
$$

un $(n-1)$-atlas. Alors, pour tout $Y \in C$ et tout morphisme $Y \longrightarrow U_{i} \times_{F} X$, on a

$$
V_{i, j} \times_{U_{i} \times{ }_{F}} Y \simeq V_{i, j} \times_{U_{i} \times X} Y .
$$

Comme $C$ a des limites finies on voit que $V_{i, j} \times_{U_{i} \times_{F} X} Y$ est représentable pour tout $j$ et tout $i$. Cela implique que pour tout $i$ et tout $j$, le morphisme

$$
V_{i, j} \longrightarrow U_{i} \times_{F} X
$$

est (-1)-représentable, et donc que $U_{i} \times_{F} X$ est 0-géométrique.

Remarque 2.6 En termes géométriques, les faisceaux (-1)-géométriques correspondent aux objets affines, les faisceaux 0-géométriques aux espaces ayant une diagonale affine, et les faisceaux 1-géométriques aux espaces sans aucune condition de séparation.

Comme dans [HAGII] on vérifie que les faisceaux géométriques sont stables par limites finies, sommes disjointes infinies et quotients par des relations d'équivalences qui sont dans $\mathbb{P}$. On renvoie à [HAGII] pour plus de détails, que nous ne reproduirons pas ici. Nous utiliserons en particulier le lemme suivant.

Lemme 2.7 Soient $X \in C$ et $F \longrightarrow X$ un morphisme dans $S h(C)$.

1. Supposons qu'il existe une famille couvrante $\left\{X_{i} \longrightarrow X\right\}$ dans $C$ telle que pour tout $i$ le faisceau $F \times_{X} X_{i}$ soit géométrique. Alors le faisceau $F$ est géométrique.

2. Supposons qu'il existe une famille couvrante $\left\{X_{i} \longrightarrow X\right\}$ dans $C$ telle que pour tout $i$ le morphisme $F \times_{X} X_{i} \longrightarrow X_{i}$ soit géométrique et dans $\mathbb{P}$. Alors le faisceau $F$ est géométrique et $F \longrightarrow X$ est dans $\mathbb{P}$.

Preuve: D'après le point (4) de la définition 2.3 on peut supposer que pour tout $i$ le morphisme $X_{i} \longrightarrow X$ est dans $\mathbb{P}$. Si pour tout $i$ on choisit un 1-atlas $\left\{U_{i, j} \longrightarrow F \times_{X} X_{i}\right\}$, alors on vérifie facilement que la famille totale $\left\{U_{i, j} \longrightarrow F\right\}$ est encore un 1-atlas. Ceci implique que $F$ est géométrique. Si de plus chaque $F \times_{X} X_{i} \longrightarrow X_{i}$ est dans $\mathbb{P}$, alors on peut choisir $U_{i, j}$ de sorte à ce que $U_{i, j} \longrightarrow X_{i}$ soit dans $\mathbb{P}$. Ceci implique que $F \longrightarrow X$ est dans $\mathbb{P}$.

Nous terminons cette section par la notion de changement de contextes géométriques. Pour cela, soient $(C, \tau, \mathbb{P})$ et $(D, \rho, \mathbb{Q})$ deux contextes géométriques. Soit $f: C \longrightarrow D$ un foncteur. 
Proposition 2.8 On suppose que le foncteur $f$ vérifie les deux conditions suivantes.

1. Le foncteur $f$ commute aux limites finies et est continu pour les topologies $\tau$ et $\rho$ (i.e. $f^{*}$ préserve les faisceaux).

2. L'image par $f$ d'un morphisme de $\mathbb{P}$ est un morphisme de $\mathbb{Q}$.

Alors le foncteur induit de la proposition 2.2

$$
f_{!}^{a}: S h(C) \longrightarrow S h(D)
$$

préserve les faisceaux géométriques et envoie les morphismes de $\mathbb{P}$ dans des morphismes de $\mathbb{Q}$.

Preuve: Commençons par montrer que $f_{!}^{a}$ préserve les morphismes $n$-représentables et envoie les morphismes $n$-représentables et dans $\mathbb{P}$ dans des morphismes $n$-représentables et dans $\mathbb{Q}$. Nous procèderons par récurrence sur $n$. Pour $n=-1$ c'est la formule

$$
f_{!}^{a}\left(h_{X}\right) \simeq h_{f(X)}
$$

et les conditions sur le foncteur $f$. Supposons que tout cela soit vrai pour $m<n$, et soit $g: F \longrightarrow G$ un morphisme $n$-représentable dans $S h(C)$. Soit $Y \in D$ et $Y \longrightarrow f_{!}^{a}(G)$ un morphisme dans $S h(D)$.

Lemme 2.9 Il existe un famille d'objets $\left\{X_{i}\right\}_{i \in I}$ dans $C$, une famille couvrante $\left\{Y_{j} \longrightarrow Y\right\}_{j \in J}$ dans $D$, une application $u: J \rightarrow I$, un morphisme

$$
\coprod_{i} X_{i} \longrightarrow G
$$

et pour tout $j$ un diagramme commutatif dans $S h(D)$

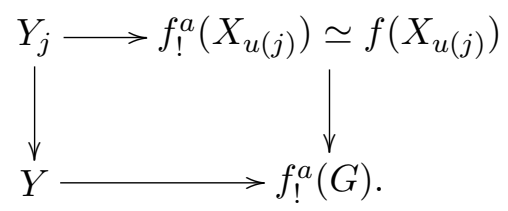

Preuve du lemme 2.9: Le foncteur $f_{!}^{a}$ préserve les épimorphismes de faisceaux, car il commute aux limites finies et aux colimites arbitraires. Soit donc $\coprod_{i} X_{i} \longrightarrow G$ un épimorphisme dans $S h(C)$ avec $X_{i}$ des objets de $C$. Alors le morphisme induit

$$
\coprod_{i} f\left(X_{i}\right) \longrightarrow f_{!}^{a}(G)
$$

est un épimorphisme dans $S h(D)$, ce qui implique le lemme par définition des épimorphismes.

Revenons à la preuve de la proposition 2.8. Il nous faut montrer que $f_{!}^{a}(F) \times_{f_{!}^{a}(G)} Y$ est un faisceau $n$-géométrique. Le lemme précédent et le lemme 2.7 permettent de supposer que le 
morphisme $Y \longrightarrow f_{!}^{a}(G)$ se factorise par un morphisme $f_{!}^{a}(X) \longrightarrow f_{!}^{a}(G)$ pour un $X \in C$ et un morphisme $Y \longrightarrow f_{!}^{a}(X)$. Mais dans ce cas, on a

$$
f_{!}^{a}(F) \times_{f_{!}^{a}(G)} Y \simeq f_{!}^{a}(F) \times_{f_{!}^{a}(G)} f_{!}^{a}(X) \times_{f_{!}^{a}(X)} Y \simeq f_{!}^{a}\left(F \times_{G} X\right) \times_{f(X)} Y .
$$

On est ainsi ramenés à démontrer que $f_{!}^{a}$ préserve les faisceaux $n$-géométriques, mais cela se déduit immédiatement de l'hypothèse de récurrence.

Il nous reste à voir que si de plus le morphisme $g$ est dans $\mathbb{P}$ alors $f_{!}^{a}(g)$ est dans $\mathbb{Q}$. Le même argument montre alors que l'on peut supposer que $G=X$ est un objet de $C$. Dans ce cas cela se déduit encore des hypothèses de récurrence.

\subsection{Analytification des espaces algébriques}

Nous allons maintenant spécifier deux contextes géométriques, le contexte algébrique et le contexte analytique (tous deux au-dessus des nombres complexes).

Nous commencerons par le contexte algébrique. Pour cela nous définissons un contexte géométrique $(C, \tau, \mathbb{P})$, pour lequel $C:=A f f$ est la catégorie des $\mathbb{C}$-schémas affines et de type fini, $\tau:=e t$ est la topologie étale, et $\mathbb{P}$ est la classe des morphismes étales. Ces notions définissent un contexte géométrique (Aff, et, et) au sens de la définition 2.3.

Définition 2.10 1. Un espace algébrique est un faisceau géométrique pour le contexte (Aff, et, et) défini ci-dessus.

2. La sous-catégorie pleine de Sh(Aff) formée des espaces algébriques sera notée Esp ${ }^{\text {alg }}$.

Remarque 2.11 Tels que nous les avons définis ci-dessus les espaces algébriques seront toujours localement de type fini sur $\mathbb{C}$. Cela provient du choix du contexte $(A f f$, et, et) pour lequel nous nous restreignons aux schémas affines de type fini. Il existe une notion plus générale d'espaces algébriques non-nécessairement localement de type fini en considérant tous les schémas affines (voir [HAGII]). Cette notion plus générale ne sera pas utilisée dans ce travail.

Passons maintenant à la description du contexte analytique $(C, \tau, \mathbb{P})$. Pour cela nous posons $C:=$ Ste, la catégorie des espaces analytiques de $\operatorname{Stein}^{1}, \tau:=$ top la topologie usuelle sur les espaces analytiques complexes, et pour $\mathbb{P}$ nous prenons la classe des morphismes étales entre espaces analytiques (i.e. des isomorphismes biholomorphes locaux). Ces notions définissent un contexte géométrique (Ste, top, et) au sens de la définition 2.3 .

Définition 2.12 1. Un espace analytique est un faisceau géométrique pour le contexte (Ste, top, et) défini ci-dessus.

2. La sous-catégorie pleine de Sh(Ste) formée des espaces analytiques sera notée Esp ${ }^{a n}$.

\footnotetext{
${ }^{1}$ Dans ce travail, un espace analytique $X$ est de Stein s'il est dénombrable à l'infini et si $H^{i}(X, \mathcal{F})=0$ pour tout $i>0$ et tout faisceau cohérent $\mathcal{F}$.
} 
Remarque 2.13 Les définitions d'espaces algébriques et d'espaces analytiques ci-dessus sont plus générales que celles que l'on rencontre usuellement dans la littérature. Par exemple, nos espaces algébriques n'ont pas des diagonales quasi-compactes alors que cette hypothèse est souvent incluse dans la définition d'espaces algébriques (cf. [Kn]). De même, nos espaces analytiques sont plus généraux que les espaces analytiques usuels. Par exemple, si $\Gamma$ est un groupe discret qui opère sans points fixes, mais non proprement, sur un espace analytique $X$, alors le faisceau quotient $X / \Gamma$ est un espace analytique au sens de la définition précédente. Cependant, ce quotient peut ne pas exister en tant qu'espace analytique au sens usuel (e.g. au sens de [Ba-St, Gr-Re]) . De plus, même lorsqu'un quotient $X / / \Gamma$ existe en tant qu'espace analytique au sens usuel, en général $X / \Gamma$ et $X / / \Gamma$ ne sont pas isomorphes. Le lecteur pourra garder en tête l'exemple suivant: $X=\mathbb{C}^{\times}$, et $\Gamma=\mathbb{Z}$ qui opère par $z \mapsto q . z$ avec $|q|=1$ et qui n'est pas une racine de l'unité. Dans ce cas $X / / \Gamma$ est réduit à un point mais $X / \Gamma$ ne l'est pas.

Considérons maintenant le foncteur d'analytification (voir [SGA1, Exp. XII])

$$
\begin{array}{ccc}
\text { Aff } & \longrightarrow \text { Ste } \\
X & \mapsto X^{a n} .
\end{array}
$$

Ce foncteur possède toutes les propriétés de la proposition 2.8, et ainsi donne lieu à un foncteur au niveau des faisceaux

$$
(-)^{a n}: S h(A f f) \longrightarrow S h(S t e) .
$$

Ce foncteur commute aux limites finies, envoie les espaces algébriques sur les espaces analytiques et préserve les morphismes étales. Nous disposons donc d'un foncteur induit

$$
(-)^{a n}: E s p^{a l g} \longrightarrow E s p^{a n}
$$

Pour terminer cette section nous allons donner une description explicite de l'analytifié d'un faisceaux $F \in S h(A f f)$, tout au moins localement en chaque point. Pour cela, si $x \in X$ est un point dans un espace de Stein et si $F \in S h(S t e)$ est un faisceau nous noterons

$$
F\left(X_{x}\right):=\operatorname{Colim}_{x \in U \subset X} F(U),
$$

où la colimite est prise sur l'ensemble filtrant des ouverts de Stein de $X$ contenant $x$. En d'autres termes, $F\left(X_{x}\right)$ est la fibre du faisceau $F$ restreint au petit site des ouverts de Stein de $X$. La notation $X_{x}$ désigne le germe d'espace analytique de $X$ en $x$. Pour tout $Y \in$ Ste nous poserons aussi

$$
\operatorname{Hom}\left(X_{x}, Y\right):=\operatorname{Colim}_{x \in U \subset X} \operatorname{Hom}(U, Y),
$$

les germes de morphismes en $x$ de $X$ vers $Y$.

Soit $\mathcal{O}_{x}$ la $\mathbb{C}$-algèbre des germes de fonctions holomorphes sur $X$ au point $x$. Pour toute $\mathbb{C}$-algèbre commutative de type fini $B$, il existe une application

$$
\operatorname{Hom}\left(X_{x},(\operatorname{Spec} B)^{a n}\right) \longrightarrow \operatorname{Hom}_{\mathbb{C}-A l g}\left(B, \mathcal{O}_{x}\right),
$$

qui à $u: X_{x} \longrightarrow(\operatorname{Spec} B)^{a n}$ associe le morphisme

$$
u^{*}: \mathcal{O}\left((\operatorname{Spec} B)^{a n}\right) \longrightarrow \mathcal{O}\left(X_{x}\right)=\mathcal{O}_{x}
$$


composé à droite avec le morphisme naturel

$$
B \longrightarrow \mathcal{O}\left((\operatorname{Spec} B)^{a n}\right)
$$

(qui à un élément de $B$ associe la fonction holomorphe sur (Spec $B)^{a n}$ correspondante).

Lemme 2.14 L'application ci-dessus

$$
\operatorname{Hom}\left(X_{x},(\operatorname{Spec} B)^{a n}\right) \longrightarrow \operatorname{Hom}_{\mathbb{C}-A l g}\left(B, \mathcal{O}_{x}\right)
$$

est bijective.

Preuve du lemme: Il est facile de voir que l'ensemble des $\mathbb{C}$-algèbres $B$ pour lesquelles la conclusion du lemme est vérifiée est stable par colimites finies. Il nous suffit donc de vérifier le lemme pour $B=\mathbb{C}[X]$, qui est alors évident.

On considère maintenant l'ensemble filtrant des sous- $\mathbb{C}$-algèbres de type fini $A \subset \mathcal{O}_{x}$. Notons $f: A f f \longrightarrow$ Ste le foncteur d'analytification, et $f_{!}: \operatorname{Pr}($ Aff $) \longrightarrow \operatorname{Pr}($ Ste $)$ le foncteur induit sur les préfaisceaux. Comme ci-dessus, on pose pour tout $F \in \operatorname{Pr}($ Ste $)$

$$
F\left(X_{x}\right):=\operatorname{Colim}_{x \in U \subset X} F(U) .
$$

Le lemme précédent implique que pour toute sous- $\mathbb{C}$-algèbre de type finie $A \subset \mathcal{O}_{x}$ on dispose d'un germe de morphisme naturel

$$
X_{x} \longrightarrow(\operatorname{Spec} A)^{a n}
$$

Pour $F \in \operatorname{Pr}(A f f)$, on dispose donc d'un morphisme naturel

$$
f_{!}(F)\left((\operatorname{Spec} A)^{a n}\right) \longrightarrow f_{!}(F)\left(X_{x}\right) .
$$

En composant avec le morphisme d'analytification

$$
F(\operatorname{Spec} A)=\operatorname{Hom}(\operatorname{Spec} A, F) \longrightarrow f_{!}(F)\left((\operatorname{Spec} A)^{a n}\right)=H o m\left(f_{!}(\operatorname{Spec} A), f_{!}(F)\right)
$$

on en déduit un morphisme

$$
F(\operatorname{Spec} A) \longrightarrow f_{!}(F)\left(X_{x}\right)
$$

fonctoriel en $A \subset \mathcal{O}_{x}$. Cela définit un morphisme

$$
\phi: \operatorname{Colim}_{A \subset \mathcal{O}_{x}} F(\operatorname{Spec} A) \longrightarrow f_{!}(F)\left(X_{x}\right) .
$$

Proposition 2.15 Avec les notations ci-dessus le morphisme

$$
\phi: \operatorname{Colim}_{A \subset \mathcal{O}_{x}} F(\operatorname{Spec} A) \longrightarrow f_{!}(F)\left(X_{x}\right)
$$

est bijectif. 
Preuve: Nous dirons qu'un préfaisceau $F \in \operatorname{Pr}(A f f)$ à la propriété $P$, si le morphisme de la proposition

$$
\phi: \operatorname{Colim}_{A \subset \mathcal{O}_{x}} F(\operatorname{Spec} A) \longrightarrow f_{!}(F)\left(X_{x}\right)
$$

est bijectif (le couple $(X, x)$ étant fixé). Il est facile de voir que la sous-catégorie pleine de $\operatorname{Pr}(A f f)$ formée des préfaisceaux ayant la propriété $P$ est stable par colimites et limites finies. Comme tout préfaisceau est colimite de représentables, on voit qu'il nous suffit de montrer que les représentables possèdent la propriété $P$. Soit donc $F=\operatorname{Spec} B$, avec $B$ une $\mathbb{C}$-algèbre de type finie. Le préfaisceau $F$ s'écrit alors comme un produit fibré de la forme

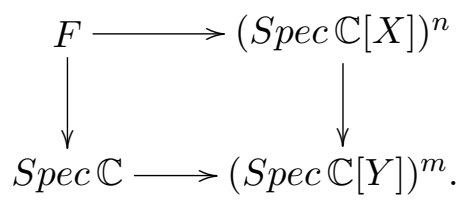

Comme l'ensemble des préfaisceaux possèdant la propriété $P$ est stable par limites finies on est ramenés au cas où $F=\mathbb{A}^{1}=\operatorname{Spec} \mathbb{C}[X]$ pour lequel la proposition est évidente.

Supposons maintenant que $F \in S h(A f f)$ soit un faisceau. Le morphisme de faisceautisation

$$
f_{!}(F) \longrightarrow a\left(f_{!}(F)\right)=F^{a n}
$$

induit alors une bijection $f_{!}\left(X_{x}\right) \simeq F^{a n}\left(X_{x}\right)$. Le morphisme $\phi$ de la proposition 2.15 peut donc aussi être considéré comme une bijection

$$
\phi: \operatorname{Colim}_{A \subset \mathcal{O}_{x}} F(\operatorname{Spec} A) \simeq F^{a n}\left(X_{x}\right) .
$$

C'est sous cette dernière forme que nous utiliserons la proposition 2.15.

\section{Catégories dérivées des espaces analytiques}

Pour un espace analytique $X$ (voir nos conventions à la section $\S 2$ ) pour la notion d'espace analytique que nous utilisons), nous disposons de sa catégorie des $\mathcal{O}_{X}$-modules $\operatorname{Mod}\left(\mathcal{O}_{X}\right)$. C'est une catégorie de Grothendieck, et nous noterons $D(X)$ sa catégorie dérivée non-bornée (voir l'appendice $\mathrm{C}$ ). La sous-catégorie pleine de $D(X)$ formée des complexes à cohomologie cohérente sera notée $D_{c o h}(X)$.

Définition 3.1 Un objet $E \in D(X)$ est parfait (nous dirons aussi complexe parfait) s'il est localement sur $X$ isomorphe à un complexe borné de $\mathcal{O}_{X}$-modules localement libres de rangs finis.

La sous-catégorie pleine des objets parfaits dans $D(X)$ sera notée $D_{\text {parf }}(X)$.

Nous avons bien entendu des inclusions

$$
D_{\text {parf }}(X) \subset D_{\text {coh }}(X) \subset D(X) .
$$

La catégorie $D(X)$ est munie d'une structure tensorielle $-\otimes^{\mathbb{L}}-$ (voir l'appendice C). La sous-catégorie $D_{\text {parf }}(X)$ est stable par $-\otimes^{\mathbb{L}}-$ et hérite donc elle aussi d'une structure tensorielle. 
Soit maintenant $f: X \longrightarrow Y$ un morphisme d'espaces analytiques. Il induit une adjonction

$$
f^{*}: \operatorname{Mod}\left(\mathcal{O}_{Y}\right) \rightleftarrows \operatorname{Mod}\left(\mathcal{O}_{X}\right): f_{*} .
$$

Comme il est expliqué dans l'appendice $\mathrm{C}$ cette adjonction se dérive alors en une adjonction au niveau des catégories dérivées

$$
\mathbb{L} f^{*}: D(Y) \rightleftarrows D(X): \mathbb{R} f_{*} .
$$

Le foncteur $\mathbb{L} f^{*}$ est naturellement compatible avec les structures tensorielles.

Proposition 3.2 Soit $f: X \longrightarrow Y$ un morphisme propre et plat d'espaces analytiques. Alors $\mathbb{L} f^{*}$ et $\mathbb{R} f_{*}$ préservent les objets parfaits et induisent une adjonction

$$
\mathbb{L} f^{*}: D_{\text {parf }}(Y) \rightleftarrows D_{\text {parf }}(X): \mathbb{R} f_{*} .
$$

Preuve: Que $\mathbb{L} f^{*}$ préserve les parfaits est clair, et ne demande d'ailleurs pas que $f$ soit propre ou plat. Il nous faut donc montrer que $\mathbb{R} f_{*}$ préserve les complexes parfaits. Nous allons déduire ceci des énoncés de finitude et de changement de bases pour les faisceaux analytiques cohérents.

Lemme 3.3 Pour tout complexe parfait $E$ sur $X$ le complexe $\mathbb{R} f_{*}(E)$ est à cohomologie cohérente et localement bornée sur $Y$.

Preuve du lemme 3.3: Pour $E$ un faisceau cohérent le lemme se déduit de [Ba-St, Thm. 4.1] (prendre $\mathcal{M}=\mathcal{O}_{Y}$ ). Par récurrence sur le nombre de faisceaux de cohomologie non-nuls on déduit alors le lemme pour tout complexe $E$ à cohomologie cohérente et bornée. Par propreté on en déduit le lemme pour tout complexe $E$ à cohomologie cohérente et localement bornée. En particulier il est vrai pour les complexes parfaits.

Lemme 3.4 Pour tout complexe parfait $E$ sur $X$, et tout faisceau cohérent $F$ sur $Y$, le morphisme naturel

$$
\mathbb{R} f_{*}(E) \otimes^{\mathbb{L}} F \longrightarrow \mathbb{R} f_{*}\left(E \otimes \mathbb{L} \mathbb{L} f^{*}(F)\right)
$$

est un isomorphisme sur $Y$.

Preuve du lemme 3.4: Comme pour le lemme 3.3 on se ramène à démontrer l'assertion du lemme pour un faisceau cohérent $E$ sur $X$. De plus, l'assertion étant locale sur $Y$, on peut supposer que le faisceau $F$ possède une résolution par des $\mathcal{O}_{Y}$-libres de rangs finis

$$
\text { . } F_{n} \longrightarrow F_{n-1} \longrightarrow \cdots \quad F_{1} \longrightarrow F_{0} \longrightarrow F
$$

(on peut par exemple remplacer $Y$ par un recouvrement par des ouverts de Stein relativement compacts dans des voisinages ouverts de Stein). On peut aussi supposer d'après le lemme 3.3 que $\mathbb{R} f_{*}(E)$ est à cohomologie bornée sur $Y$.

Soit $p \in \mathbb{Z}$. Nous affirmons qu'il existe un entier $m$, tel que le morphisme naturel

$$
F_{*}[\leq m] \longrightarrow F
$$


induise un isomorphisme sur les faisceaux de cohomologie

$$
\begin{gathered}
\underline{H}^{p}\left(\mathbb{R} f_{*}(E) \otimes F_{*}[\leq m]\right) \longrightarrow \underline{H}^{p}\left(\mathbb{R} f_{*}(E) \otimes F_{*}\right) \\
\underline{H}^{p}\left(\mathbb{R} f_{*}\left(E \otimes f^{*}\left(F_{*}[\leq m]\right)\right)\right) \longrightarrow \underline{H}^{p}\left(\mathbb{R} f_{*}\left(E \otimes f^{*}\left(F_{*}\right)\right)\right),
\end{gathered}
$$

où $F_{*}[\leq m]$ est la résolution tronquée définie par

$$
\left(F_{*}[\leq m]\right)_{n}=F_{n} \text { si } n \leq m \text { et } 0 \text { sinon. }
$$

Ces deux assertions se déduisent aisément du fait que $\mathbb{R} f_{*}(E)$ soit un complexe à cohomologie bornée sur $Y$. Le diagramme commutatif dans $D(Y)$

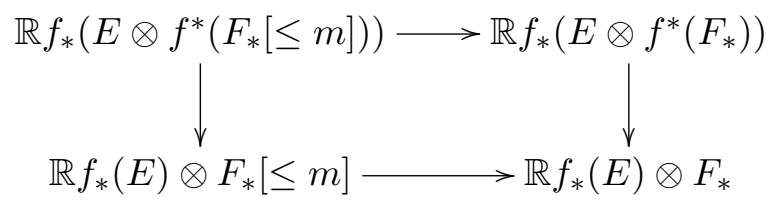

montre alors qu'il suffit de démontrer que pour tout $m$ le morphisme naturel

$$
\mathbb{R} f_{*}\left(E \otimes f^{*}\left(F_{*}[\leq m]\right)\right) \longrightarrow \mathbb{R} f_{*}(E) \otimes F_{*}[\leq m]
$$

est un isomorphisme dans $D(Y)$. Mais cela se ramène facilement par récurrence sur $m$ au cas où $m=0$ qui est évident.

Nous pouvons maintenant démontrer la proposition 3.2. Comme l'assertion est locale sur $Y$ on peut utiliser le lemme 3.3 et supposer que $\mathbb{R} f_{*}(E)$ est à cohomologie bornée sur $Y$. On applique alors le lemme 3.4 à $F=k(y)$, le faisceau gratte-ciel en un point $y \in Y$. Comme $f$ est plat on a $\mathbb{L} f^{*}(k(y)) \simeq \mathcal{O}_{X_{y}}$, où $X_{y}$ est la fibre de $f$ en $y$.

Cela nous dit que le morphisme naturel

$$
\mathbb{R} f_{*}(E) \otimes^{\mathbb{L}} k(y) \longrightarrow \mathbb{R} f_{*}\left(E \otimes^{\mathbb{L}} \mathcal{O}_{X_{y}}\right)
$$

est un quasi-isomorphisme. Or, le second membre est quasi-isomorphe au complexe d'hypercohomologie de $X_{y}$ à coefficient dans le complexe parfait $E \otimes^{\mathbb{L}} \mathcal{O}_{X_{y}}$. Ceci implique donc que pour tout $y \in Y$, le complexe $\mathbb{R} f_{*}(E) \otimes^{\mathbb{L}} k(y)$ est cohomologiquement borné.

Lemme 3.5 Soit $F$ un complexe sur $Y$ à cohomologie cohérente et bornée. Soit $y \in Y$ tel que le complexe $F \otimes^{\mathbb{L}} k(y)$ soit cohomologiquement borné. Alors il existe un voisinage $Y^{\prime}$ de y tel que la restriction de $F$ sur $Y^{\prime}$ soit un complexe parfait.

Preuve du lemme 3.5: Quitte à restreindre $F$ à un voisinage de $y$ on peut supposer que $F$ est quasi-isomorphe à un complexe $F_{*}^{\prime}$ de $\mathcal{O}_{Y}$-modules libres de rangs finis borné à droite. Supposons alors que $H^{i}\left(F \otimes^{\mathbb{L}} k(y)\right)=0$ pour tout $i<n$, pour $n \leq 0$. Soit $\tau_{\geq n} F_{*}^{\prime}$ le tronqué de $F_{*}^{\prime}$ (par définition la cohomologie de $\tau_{\geq n} F_{*}^{\prime}$ coincide avec celle de $F_{*}^{\prime}$ en degrés supérieurs à $n$ et est nulle ailleurs). On a donc

$$
\left(\tau_{\geq n} F_{*}^{\prime}\right)_{n}:=\operatorname{Coker}\left(F_{n-1}^{\prime} \longrightarrow F_{n}^{\prime}\right) .
$$


Par hypothèse on trouve que

$$
\operatorname{Tor}_{i}^{\mathcal{O}_{Y}}\left(\left(\tau_{\geq n} F_{*}^{\prime}\right)_{n}, k(y)\right)=0
$$

pour tout $i>0$. Ceci implique donc que $\left(\tau_{\geq n} F_{*}^{\prime}\right)_{n}$ est localement libre dans un voisinage de $y$. Quitte à restreindre $Y$, on voit donc que le complexe $\tau_{\geq n} F_{*}^{\prime}$ est parfait, et de plus que le morphisme naturel

$$
F_{*}^{\prime} \longrightarrow \tau_{\geq n} F_{*}^{\prime}
$$

induit un quasi-isomorphisme

$$
F_{*}^{\prime} \otimes^{\mathbb{L}} k(y) \longrightarrow \tau_{\geq n} F_{*}^{\prime} \otimes^{\mathbb{L}} k(y) .
$$

Ceci implique alors que le cône $C$ du morphisme $F_{*}^{\prime} \longrightarrow \tau_{\geq n} F_{*}^{\prime}$ est tel que $C \otimes^{\mathbb{L}} k(y)$ soit contractile. Comme $C$ est un complexe à cohomologie cohérente et bornée cela implique par Nakayama que $C$ est quasi-isomorphe à 0 dans un voisinage de $Y$. Ainsi, $F_{*}^{\prime} \longrightarrow \tau_{\geq n} F_{*}^{\prime}$ est un quasi-isomorphisme dans un voisinage de $y$, ce qui implique que $F$ est parfait dans un voisinage de $y$.

Le lemme 3.5 appliqué au complexe $\mathbb{R} f_{*}(E)$ termine alors la preuve de la proposition.

On déduit de la proposition et de sa preuve la formule du changement de bases suivante.

\section{Corollaire 3.6 Soit}

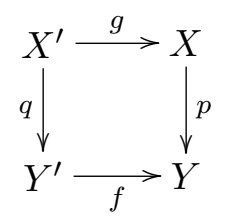

un diagramme cartésien d'espaces analytiques avec $p$ un morphisme propre et plat. Alors, pour tout complexe parfait $E$ sur $X$, le morphisme naturel

$$
\mathbb{L} f^{*} \mathbb{R} p_{*}(E) \longrightarrow \mathbb{R} q_{*} \mathbb{L} g^{*}(E)
$$

est un isomorphisme dans $D\left(Y^{\prime}\right)$.

Preuve: Tout d'abord, le lemme 3.4 appliqué à $F=k(y)$, le faisceau gratte-ciel en un point $y \in Y$, implique que le corollaire 3.6 est vrai lorsque $Y^{\prime}$ est un point. Les objets $\mathbb{L} f^{*} \mathbb{R} p_{*}(E)$ et $\mathbb{R} q_{*} \mathbb{L} g^{*}(E)$ étant parfaits sur $Y^{\prime}$ (d'après la proposition 3.2 ), le morphisme

$$
\mathbb{L} f^{*} \mathbb{R} p_{*}(E) \longrightarrow \mathbb{R} q_{*} \mathbb{L} g^{*}(E)
$$

est un isomorphisme dans $D\left(Y^{\prime}\right)$ si et seulement si pout tout $y^{\prime} \in Y^{\prime}$ d'inclusion $i_{y^{\prime}}:\left\{y^{\prime}\right\} \hookrightarrow Y^{\prime}$, le morphisme induit

$$
\mathbb{L} i_{y^{\prime}}^{*} \mathbb{L} f^{*} \mathbb{R} p_{*}(E) \longrightarrow \mathbb{L} i_{y^{\prime}}^{*} \mathbb{R} q_{*} \mathbb{L} g^{*}(E)
$$

est un isomorphisme dans $D\left(\left\{y^{\prime}\right\}\right)$. Ainsi, les formules de changement de bases pour $\left\{y^{\prime}\right\} \longrightarrow Y^{\prime}$ et $\left\{y^{\prime}\right\} \longrightarrow Y^{\prime}$ impliquent le corollaire. 
Nous terminons cette section par la définition de la version dg des catégories dérivées. Pour cela nous rappelons l'existence de la catégorie de modèles $C\left(\mathcal{O}_{X}\right)$ des complexes de $\mathcal{O}_{X}$-modules sur un espace analytique $X$ (voir l'appendice $\mathrm{C}$ ). Cette catégorie de modèles est une $C(\mathbb{C})$ catégorie de modèles au sens de [Ho, 4.2.18], où $C(\mathbb{C})$ est la catégorie de modèles monoïdale des complexes de $\mathbb{C}$-espaces vectoriels (pour laquelle les équivalences sont les quasi-isomorphismes et les fibrations sont les épimorphismes). Nous considérons alors la dg-catégorie $\operatorname{Int}\left(C\left(\mathcal{O}_{X}\right)\right.$, des objets fibrants et cofibrants dans $C\left(\mathcal{O}_{X}\right)$ (voir [To1] pour plus de détails sur la construction Int).

Définition 3.7 $L a$ dg-catégorie dérivée des $\mathcal{O}_{X}$-modules est

$$
L(X):=\operatorname{Int}\left(C\left(\mathcal{O}_{X}\right)\right) .
$$

$L a$ dg-catégorie dérivée des $\mathcal{O}_{X}$-modules parfaits est la sous-dg-catégorie pleine de $L(X)$ formée des complexes parfaits.

\section{Analytification des espaces de modules d'objets dans des dg- catégories}

Dans cette section nous rappelons l'existence d'un espace algébrique $M(B)$, classifiant les $B$-dgmodules simples sur une dg-algèbre $B$ propre et lisse. Nous expliciterons aussi le foncteur des points de l'espace analytique $M(B)^{a n}$. Nous supposerons le lecteur familier avec les notions de dg-algèbres, dg-modules et de lissité et propreté, telles que présentées par exemple dans [To-Va].

\subsection{Rappels sur les espaces de modules de dg-modules simples}

Considérons une dg-algèbre $B$ sur $\mathbb{C}$. On supposera que $B$ est propre est lisse, c'est-à-dire qu'elle vérifie les deux conditions suivantes (voir e.g. [Ko-So, To-Va]).

1. Le complexe sous-jacent à $B$ est parfait (i.e. $B$ est à cohomologie bornée et de dimension finie).

2. $B$ est un objet compact dans $D\left(B \otimes B^{o p}\right)$, la catégorie dérivée des $B \otimes B^{o p}$-dg-modules.

Pour toute $\mathbb{C}$-algèbre commutative $A$, non nécessairement de type fini, on dispose d'une nouvelle $\mathbb{C}$-dg-algèbre $B \otimes A$ et de sa catégorie dérivée $D(B \otimes A)$. Si $A \longrightarrow A^{\prime}$ est un morphisme de $\mathbb{C}$-algèbres commutatives, le mophisme $B \otimes A \longrightarrow B \otimes A^{\prime}$ induit un foncteur de changement de bases

$$
-\otimes_{A}^{\mathbb{L}} A^{\prime}=-\otimes_{B \otimes A}^{\mathbb{L}} B \otimes A^{\prime}: D(B \otimes A) \longrightarrow D\left(B \otimes A^{\prime}\right) .
$$

La catégorie $B \otimes A-M o d$, des $B \otimes A$-dg-modules, possède un enrichissement naturel dans la catégorie monoïdale $C(A)$ des complexes de $A$-modules. En effet, pour $E \in B \otimes A-M o d$ et $L \in C(A)$, on forme $L \otimes_{A} E$ qui est encore un $B \otimes A$-dg-module en utilisant la structure de $B$-dg-module sur $E$ et celle de $A$-dg-module sur $L$. En clair, l'action de $B \otimes A$ sur $L \otimes_{A} E$ est donnée par le morphisme

$$
(B \otimes A) \otimes\left(L \otimes_{A} E\right) \simeq(A \otimes L) \otimes_{A}(B \otimes E) \stackrel{\mu_{L} \otimes \mu_{E}}{\longrightarrow} L \otimes_{A} E,
$$


où $\mu_{L}$ et $\mu_{E}$ sont les actions de $A$ et $B$ sur $L$ et $E$. Cet enrichissement de $B \otimes A-M o d$ dans $C(A)$ fait de $B \otimes A$ une $C(A)$-catégorie de modèles au sens de [Ho, 4.2.18] (on peut aussi voir cela en remarquant que $B \otimes A-M o d$ est aussi la catégorie des $B \otimes A$-modules dans $C(A)$, lorsque $B \otimes A$ est vu comme un monoïde dans $C(A)$ (i.e. comme une $A$-dg-algèbre)). De ceci nous déduisons l'existence d'un enrichissement de $D(B \otimes A)$ dans $D(A)$. En particulier, on dispose de Hom à valeurs dans $D(A)$ que nous noterons

$$
\mathbb{R}_{\operatorname{Hom}}(-,-): D(B \otimes A)^{o p} \times D(B \otimes A) \longrightarrow D(A) .
$$

Définition 4.1 1. Un objet $E \in D(B \otimes A)$ est parfait si le complexe de A-modules sousjacent à $E$ est un complexe parfait.

2. un objet $E \in D(B \otimes A)$ est rigide si pour tout morphisme de $\mathbb{C}$-algèbres commutatives $A \longrightarrow A^{\prime}$, et pour tout $i<0$ on a

$$
\operatorname{Ext}^{i}\left(E \otimes_{A}^{\mathbb{L}} A^{\prime}, E \otimes_{A}^{\mathbb{L}} A^{\prime}\right)=0,
$$

où les Ext sont calculés dans $D\left(B \otimes A^{\prime}\right)$.

3. Un objet $E \in D(B \otimes A)$ est simple s'il est rigide et si de plus pour tout morphisme de $\mathbb{C}$-algèbres commutatives $A \longrightarrow A^{\prime}$ le morphisme naturel

$$
A^{\prime} \longrightarrow \operatorname{Ext}^{0}\left(E \otimes_{A}^{\mathbb{L}} A^{\prime}, E \otimes_{A}^{\mathbb{L}} A^{\prime}\right)
$$

est un isomorphisme.

Remarque 4.2 La notion d'objet parfait ci-dessus correspond en réalité à la notion d'objet pseudo-parfait de [To-Va]. Cependant, comme $B$ est propre est lisse on sait que les objets pseudo-parfaits sont exactement les objets parfaits (voir [To-Va, Lem. 2.8 (3)] pour une preuve de ce fait).

Nous aurons besoin des quelques résultats suivants.

Proposition 4.3 Soient $A$ une $\mathbb{C}$-algèbre commutative et écrivons $A=\operatorname{Colim}_{i} A_{i}$, où la colimite est prise sur l'ensemble filtrant des sous-C-Clgèbres de type fini $A_{i} \subset A$.

1. Le foncteur naturel

$$
\operatorname{Colim}\left(-\otimes_{A}^{\mathbb{L}} A_{i}\right): \operatorname{Colim} D\left(B \otimes A_{i}\right) \longrightarrow D(B \otimes A)
$$

induit une équivalence sur les catégories d'objets parfaits

$$
C \operatorname{Colim} D_{\text {parf }}\left(B \otimes A_{i}\right) \simeq D_{\text {parf }}(B \otimes A) .
$$

2. Soit $E_{i} \in D\left(B \otimes A_{i}\right)$ un objet parfait. Alors $E_{i} \otimes_{A_{i}}^{\mathbb{L}} A \in D(B \otimes A)$ est simple si et seulement s'il existe $A_{i} \subset A_{j} \subset A$, avec $A_{j}$ de type fini et telle que $E_{i} \otimes \mathbb{L}_{A_{i}} A_{j} \in D\left(B \otimes A_{j}\right)$ soit simple. 
Preuve: Le point (1) est une conséquence de [To-Va, Lem. 2.10]. La suffisance de la condition dans (2) se déduit des définitions, car être simple est clairement stable par changement de bases. Il nous reste à montrer la nécessité. Pour cela, supposons que $E:=E_{i} \otimes_{A_{i}}^{\mathbb{L}} A$ soit simple dans $D(B \otimes A)$. On pose $E_{j}:=E_{i} \otimes \mathbb{L}_{A_{i}} A_{j}$ pour tout $j$ tel que $A_{i} \subset A_{j}$ (nous dirons alors que $j \geq i$ ).

Pour tout $j \geq i$, on dispose d'un morphisme de complexes de $A_{j}$-modules

$$
A_{j} \longrightarrow \mathbb{R} \underline{\operatorname{Hom}}_{B}\left(E_{j}, E_{j}\right),
$$

où le membre de droite fait référence à l'enrichissement naturel de $D\left(B \otimes A_{j}\right)$ dans $D\left(A_{j}\right)$. Comme le complexe de $A$-modules sous-jacent à $B \otimes A$ est parfait et que $E_{j}$ est un $B \otimes A$-dgmodule parfait on voit que le complexe de $A$-modules $\mathbb{R} \underline{\operatorname{Hom}}_{B}\left(E_{j}, E_{j}\right)$ est parfait. De plus, le fait que $E_{j}$ soit parfait implique aussi que le morphisme naturel

$$
\mathbb{R} \underline{\operatorname{Hom}}_{B}\left(E_{i}, E_{i}\right) \otimes \underline{\mathbb{L}}_{i} A_{j} \longrightarrow \mathbb{R} \underline{\operatorname{Hom}}_{B}\left(E_{j}, E_{j}\right)
$$

est un quasi-isomorphisme. On déduit de cela et du point (1) que le morphisme naturel

$$
\mathbb{R} \underline{\operatorname{Hom}}_{B}\left(E_{i}, E_{i}\right) \otimes_{A_{i}}^{\mathbb{L}} A \simeq \operatorname{colim}_{j \geq i} \mathbb{R} \underline{\operatorname{Hom}}_{B}\left(E_{i}, E_{i}\right) \otimes_{A_{i}}^{\mathbb{L}} A_{j} \longrightarrow \mathbb{R} \underline{\operatorname{Hom}}_{B}(E, E)
$$

est un quasi-isomorphisme.

On considère maintenant le morphisme naturel

$$
A \longrightarrow \mathbb{R} \underline{\operatorname{Hom}}_{B}(E, E),
$$

qui est un morphisme de complexes parfaits de $A$-modules. On note $K$ son cône. D'après ce que l'on a vu, on a $K \simeq K_{i} \otimes \mathbb{L}_{i} A$, où $K_{i}$ est le cône du morphisme

$$
A_{i} \longrightarrow \mathbb{R} \underline{\operatorname{Hom}}_{B}\left(E_{i}, E_{i}\right) \text {. }
$$

Par hypothèse $E$ est simple, ce qui est équivalent au fait que $K$ soit de Tor amplitude contenue dans $] 0, \infty[$.

Lemme 4.4 Soit $A=\operatorname{colim}_{i} A_{i}$ une colimite filtrante d'anneaux commutatifs et $K_{i}$ un complexe parfait de $A_{i}$-modules pour un indice $i$ donné. Si le complexe de A-modules $K_{i} \otimes_{A_{i}}^{\mathbb{L}} A$ est de Tor amplitude contenue dans $[a, b]$ alors il existe un indice $j \geq i$ tel que $K_{i} \otimes \mathbb{L}_{A_{i}} A_{j}$ soit de Tor amplitude contenue dans $[a, b]$ en tant que complexe de $A_{j}$-modules

Preuve du lemme 4.4: On supposera que $K_{i}$ est un complexe borné de $A_{i}$-modules projectifs et de rangs finis. Par hypothèse, il existe un complexe de $A$-modules projectifs de type fini $L$, avec $L^{n}=0$ pour tout $n \notin[a, b]$, et une équivalence d'homotopie $u: L \longrightarrow K$. Le complexe $L$ et le morphisme $u$ sont alors tous deux définis sur un $A_{j}$ avec $j \geq i$, et il existe donc un morphisme de complexes

$$
u_{j}: L_{j} \longrightarrow K_{i} \otimes_{A_{i}} A_{j}
$$

avec $u_{j} \otimes_{A_{j}} A=u$ (dans cette notation $L_{j}$ est un complexe de $A_{j}$-modules projectifs de type fini avec $L_{j}^{n}=0$ pour tout $n \notin[a, b]$, et tel que $\left.L_{j} \otimes_{A_{j}} A \simeq L\right)$. On peut faire de même avec un inverse à homotopie près $v: K \longrightarrow L$ de $u$. Quitte à prendre un $j$ plus grand on peut aussi trouver $v_{j}: K_{i} \otimes_{A_{i}} A_{j} \longrightarrow L_{j}$ tel que $v_{j} \otimes_{A_{j}} A=v$. Enfin, les homotopies $h$ et $k$ reliant $v u$ et 
$u v$ avec les identités sont elles aussi définissables sur un $A_{j}$ pour $j$ assez grand. Ainsi, quitte à prendre $j$ assez grand on voit que le morphisme

$$
u_{j}: L_{j} \longrightarrow K_{i} \otimes_{A_{i}} A_{j}
$$

est une équivalence d'homotopie. Cela implique que $K_{i} \otimes \mathbb{L}_{A_{i}} A_{j}$ est de Tor amplitude contenue dans $[a, b]$.

Le lemme précédent implique qu'il existe $A_{i} \subset A_{j} \subset A$ tel que $K_{i} \otimes_{A_{i}}^{\mathbb{L}} A_{j}$ doit de Tor amplitude contenue dans $] 0, \infty\left[\right.$. Comme nous avons vu que $K_{j}$ est le cône du morphisme

$$
A_{j} \longrightarrow \mathbb{R} \underline{\operatorname{Hom}}_{B}\left(E_{j}, E_{j}\right),
$$

nous en déduisons que $E_{j}$ est simple.

Corollaire 4.5 Soient $A$ une $\mathbb{C}$-algèbre commutative et écrivons $A=$ Colim $_{i} A_{i}$, où la colimite est prise sur l'ensemble filtrant des sous-C-Calgèbres de type fini $A_{i} \subset A$. Notons $D_{\text {pasi }}(B \otimes A)$ et $D_{\text {pasi }}\left(B \otimes A_{i}\right)$ les catégories d'objets parfaits et simples. Alors le foncteur naturel

$$
\operatorname{Colim} D_{\text {pasi }}\left(B \otimes A_{i}\right) \longrightarrow D_{\text {pasi }}(B \otimes A)
$$

est une équivalence.

Preuve: Découle de la proposition 4.3.

Proposition 4.6 Soit $(A, m)$ une $\mathbb{C}$-algèbre locale et noethérienne. Alors un objet parfait $E \in$ $D(B \otimes A)$ est simple si et seulement s'il satisfait aux deux conditions suivantes.

1. Pour tout $i<0$ on a

$$
\operatorname{Ext}^{i}\left(E \otimes_{A}^{\mathbb{L}} A / m, E \otimes_{A}^{\mathbb{L}} A / m\right)=0,
$$

où les Ext sont calculés dans $D(B \otimes A / m)$.

2. Le morphisme naturel

$$
A / m \longrightarrow \operatorname{Ext}^{0}\left(E \otimes_{A}^{\mathbb{L}} A / m, E \otimes_{A}^{\mathbb{L}} A / m\right)
$$

est un isomorphisme.

Preuve: Comme nous l'avons utilisé lors de la preuve de la proposition 4.3, E est simple si et seulement si le cône du morphisme

$$
A \longrightarrow \mathbb{R} \underline{\operatorname{Hom}}_{B}(E, E)
$$

est de Tor amplitude contenue dans ]0, $\infty[$. Or, comme $A$ est noethérien, on sait qu'un $A$-module de type fini $M$ est plat si et seulement si $\operatorname{Tor}_{n}^{A}(M, A / m)=0$ pour $n>0$. On déduit facilement de cela qu'un complexe parfait $K$ sur $A$ est de Tor amplitude contenue dans un intervalle $[a, b]$ 
si et seulement si $K \otimes_{A}^{\mathbb{L}} A / m$ est de Tor amplitude contenue dans $[a, b]$ (i.e. si et seulement si $\operatorname{Tor}_{n}^{A}\left(K \otimes_{A}^{\mathbb{L}} A / m\right)=0$ pour $\left.n \notin[-b,-a]\right)$. Ainsi, $E$ est simple si et seulement si $K$ est de Tor amplitude contenue dans ]0, [, et donc si et seulement si $K \otimes_{A}^{\mathbb{L}} A / m$ est de Tor amplitude contenue dans ]0, $\infty$ [. Comme $E$ est un $B \otimes A$-dg-module parfait on sait que $K \otimes_{A}^{\mathbb{L}} A / m$ est le cône du morphisme induit

$$
A / m \longrightarrow \mathbb{R} \underline{\operatorname{Hom}}_{B}\left(E \otimes_{A}^{\mathbb{L}} A / m, E \otimes_{A}^{\mathbb{L}} A / m\right) .
$$

Ce qui termine la preuve de la proposition.

Nous définissons maintenant un préfaisceau $M^{\prime}(B)$ sur Aff de la façon suivante. Pour $A$ une $\mathbb{C}$-algèbre de type finie, nous noterons $M^{\prime}(B)(A)$ l'ensemble des classes d'isomorphismes d'objets parfaits et simples dans $D(B \otimes A)$. Les changements de bases préservent les objets parfaits et simples. Ainsi, un morphisme $A \longrightarrow A^{\prime}$ entre $\mathbb{C}$-algèbres de type fini induit une application

$$
-\otimes_{A}^{\mathbb{L}} A^{\prime}: M^{\prime}(B)(A) \longrightarrow M^{\prime}(B)\left(A^{\prime}\right) .
$$

Ceci définit un préfaisceau $M^{\prime}(B) \in \operatorname{Pr}(A f f)$.

Définition 4.7 Le faisceau des B-dg-modules parfaits et simples est le faisceau associé au préfaisceau $M^{\prime}(B)$. Il sera noté

$$
M(B):=a\left(M^{\prime}(B)\right) .
$$

Un important corollaire du théorème principal de [To-Va] est la représentabilité de $M(B)$ par un espace algébrique localement de type fini.

Théorème 4.8 (Voir [To-Va, Cor. 3.22]) Le faisceau $M(B)$ est un espace algébrique au sens de la définition 2.10. Il est de plus quasi-séparé (c'est à dire que le morphisme diagonal $M(B) \longrightarrow$ $M(B) \times M(B)$ est quasi-compact).

\subsection{Description de l'espace $M(B)^{a n}$}

Comme précédemment, soit $B$ une $\mathbb{C}$-dg-algèbre propre et lisse. Dans cette section nous nous proposons de donner une description de l'espace analytique $M(B)^{a n}$.

Rappelons que pour tout espace de Stein $S$, on dispose d'une catégorie de modèles $C\left(\mathcal{O}_{S}\right)$ des complexes de $\mathcal{O}_{S}$-modules (sur le petit site des ouverts de Stein de $S$, voir l'appendice C). Cette catégorie de modèles est de plus une $C(\mathbb{C})$-catégorie de modèles. On peut donc parler de $B$-dg-modules dans $C\left(\mathcal{O}_{S}\right)$ : il s'agit d'objets $E \in C\left(\mathcal{O}_{S}\right)$ munis de morphismes

$$
m: B \otimes E \longrightarrow E
$$

qui sont unitaires et associatifs en un sens évident (le produit tensoriel $B \otimes E$ est ici pris au-dessus de $\mathbb{C}$ ). Les morphismes entre $B$-dg-modules dans $C\left(\mathcal{O}_{S}\right)$ sont les morphismes dans $C\left(\mathcal{O}_{S}\right)$ qui commutent avec les morphismes structuraux $m$. Nous noterons la catégorie des $B$-dg-modules par $B-\operatorname{Mod}(S)$.

On peut aussi voir un $B$-dg-modules dans $C\left(\mathcal{O}_{S}\right)$ comme un module sur $B \otimes \mathcal{O}_{S}$ considéré comme monoïde associatif et unitaire dans la catégorie monoïdale $\left(C\left(\mathcal{O}_{S}\right), \otimes_{\mathcal{O}_{S}}\right)$. Ainsi, [S-S] 
implique l'existence d'une structure de catégorie de modèles sur $B-\operatorname{Mod}(S)$, dont les fibrations et les équivalences sont définies dans $C\left(\mathcal{O}_{S}\right)$ par oubli de l'action de $B$. Nous noterons

$$
D(S, B):=H o(B-\operatorname{Mod}(S))
$$

la catégorie homotopique des $B$-dg-modules sur $S$. De plus, cette catégorie de modèles est naturellement enrichie dans la catégorie de modèles monoïdale $C\left(\mathcal{O}_{S}\right)$. On dispose ainsi de Hom dans $D(S, B)$ à valeurs dans $D(S)=H o\left(C\left(\mathcal{O}_{S}\right)\right)$ notés

$$
\mathbb{R}_{\operatorname{Hom}_{B}}(-,-): D(S, B)^{o p} \times D(S, B) \longrightarrow D(S) .
$$

Soit maintenant $f: S \longrightarrow S^{\prime}$ un morphisme entre espaces de Stein. On dispose d'une adjonction de Quillen

$$
f^{*}: C\left(\mathcal{O}_{S^{\prime}}\right) \rightleftarrows C\left(\mathcal{O}_{S}\right): f_{*} .
$$

Comme le foncteur $f^{*}$ est de plus muni d'une structure naturelle de foncteur monoïdal symétrique, cette adjonction induit une nouvelle adjonction de Quillen

$$
f^{*}: B-\operatorname{Mod}\left(S^{\prime}\right) \rightleftarrows B-\operatorname{Mod}(S): f_{*}
$$

sur les catégories de modèles de $B$-dg-modules. On obtient donc une adjonction dérivée

$$
\mathbb{L} f^{*}: D\left(S^{\prime}, B\right) \rightleftarrows D(S, B): \mathbb{R} f_{*} .
$$

Définition 4.9 Soit $S$ un espace de Stein.

1. Un objet $E \in D(S, B)$ est parfait si le complexe de $\mathcal{O}_{S}$-modules sous-jacent à $E$ est un complexe parfait.

2. un objet $E \in D(S, B)$ est rigide si pour tout point $i_{s}:\{s\} \hookrightarrow S$, et pour tout $i<0$ on a

$$
\operatorname{Ext}^{i}\left(\mathbb{L} i_{s}^{*}(E), \mathbb{L} i_{s}^{*}(E)\right):=0,
$$

où les groupes Ext sont calculés dans $D(\{s\}, B) \simeq D(B)$.

3. Un objet $E \in D(S, B)$ est simple s'il est rigide et si de plus pour tout point $i_{s}:\{s\} \hookrightarrow S$, le morphisme naturel

$$
\mathbb{C} \longrightarrow \operatorname{Ext}^{0}\left(\mathbb{L} i_{s}^{*}(E), \mathbb{L} i_{s}^{*}(E)\right)
$$

est un isomorphisme.

Nous définissons maintenant un foncteur

$$
F_{B}: S t e^{o p} \longrightarrow \text { Ens }
$$

de la façon suivante. Pour $S \in$ Ste, l'ensemble $F_{B}(S)$ est le sous-ensemble des classes d'isomorphismes d'objets parfaits et simples de $D(S, B)$. Pour $f: S \longrightarrow S^{\prime}$ un morphisme entre espaces de Stein, on dispose du changement de bases

$$
\mathbb{L} f^{*}: D\left(S^{\prime}, B\right) \longrightarrow D(S, B) .
$$


Par définition ce foncteur préserve les conditions objets parfaits et simples et donc induit une application

$$
\mathbb{L} f^{*}: F_{B}\left(S^{\prime}\right) \longrightarrow F_{B}(S) \text {. }
$$

Ceci définit le foncteur $F_{B}$.

Proposition 4.10 Soit $S$ un espace de Stein et $E \in D(S, B)$ un objet parfait. Supposons qu'il existe un point $s \in S$ tel que $\mathbb{L} i_{s}^{*}(E)$ soit un objet simple dans $D(\{s\}, B) \simeq D(B)$. Alors il existe un voisinage ouvert de Stein $s \in U \subset S$ tel que la restriction de $E$ à $U$ soit un objet simple dans $D(U, B)$.

Preuve: C'est le même principe que pour la proposition 4.6, on montre que l'ensemble des points $s$ de $S$ tels que $\mathbb{L} i_{s}^{*}(E)$ soit un objet simple est le lieu où un certain complexe parfait est de Tor amplitude strictement positive. Pour cela, on considère la catégorie de modèles $B-\operatorname{Mod}(S)$. On rappelle que cette catégorie est naturellement enrichie sur la catégorie monoïdale $C\left(\mathcal{O}_{S}\right)$ des complexes de $\mathcal{O}_{S}$-modules. Cet enrichissement fait de $B-\operatorname{Mod}(S)$ une $C\left(\mathcal{O}_{S}\right)$-catégorie de modèles. Les Hom enrichis de $B-\operatorname{Mod}(S)$ à valeurs dans $C\left(\mathcal{O}_{S}\right)$ seront notés $\underline{H o m}_{B}$, et leur version dérivée sera notée $\mathbb{R} \underline{H o m}_{B}$.

Avec ces notations, on dispose d'un morphisme de complexes de $\mathcal{O}_{S}$-modules

$$
\mathcal{O}_{S} \longrightarrow \mathbb{R} \underline{\operatorname{Hom}}_{B}(E, E),
$$

bien défini dans $D(S)$. Nous commençons par remarquer que $\mathbb{R} \underline{H o m}_{B}(E, E)$ est un complexe parfait sur $S$. En effet, on dispose d'un foncteur bi-exact entre catégories triangulées

$$
D\left(B \otimes B^{o p}\right) \times D(S, B) \longrightarrow D(S, B),
$$

qui à $P \in D\left(B \otimes B^{o p}\right)$ et $F \in D(S, B)$ associe $P \otimes_{B}^{\mathbb{L}} F$. Ce foncteur envoie le couple $(B, E)$ sur $E$. Or, comme $B$ est lisse, on sait que $B$ appartient à la sous-catégorie triangulée et épaisse engendrée par $B \otimes B^{o p}$. Cela implique que $E \simeq B \otimes \mathbb{L}_{B} E$ appartient à la sous-catégorie triangulée et épaisse engendrée par $\left(B \otimes B^{o p}\right) \otimes_{B}^{\mathbb{L}} E \simeq B \otimes E$. On en déduit que $E$ appartient à la souscatégorie triangulée et épaisse engendrée par les objets de la forme $B \otimes F$, où $F$ est un complexe parfait sur $S$. Ainsi, le complexe $\mathbb{R} \underline{\operatorname{Hom}}_{B}(E, E)$ appartient à la sous-catégorie triangulée et épaisse de $D(S)$ engendrée par les objets de la forme $\mathbb{R}_{H_{o m}}(B \otimes F, E)$. Or, comme $B \otimes F$ est un $B$-dg-module libre, le complexe $\mathbb{R} \underline{\operatorname{Hom}}_{B}(B \otimes F, E)$ s'identifie naturellement au complexe $\mathbb{R} \underline{\operatorname{Hom}}(F, E)$ des morphismes de $F$ vers $E$ en tant que complexes de $\mathcal{O}_{S}$-modules. Comme $E$

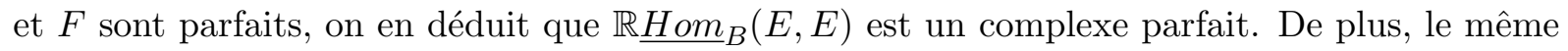
argument montre que pour tout point $s \in S$, le morphisme naturel

$$
\mathbb{L} i_{s}^{*}\left(\mathbb{R} \underline{\operatorname{Hom}}_{B}(E, E)\right) \longrightarrow \mathbb{R} \underline{\operatorname{Hom}}_{B}\left(\mathbb{L} i_{s}^{*}(E), \mathbb{L} i_{s}^{*}(E)\right)
$$

est un quasi-isomorphisme.

Revenons au morphisme

$$
\mathcal{O}_{S} \longrightarrow \mathbb{R} \underline{\operatorname{Hom}}_{B}(E, E)
$$

et notons $K$ son cône. C'est un complexe parfait, et par définition $\mathbb{L} i_{s}^{*}(E)$ est un objet simple si et seulement si $H^{i}\left(\mathbb{L} i_{s}^{*}(K)\right)=0$ pour tout $i \leq 0$. Cela est équivalent au fait qu'au voisinage du 
point $s$ le complexe $K$ est quasi-isomorphe à un complexe borné de fibrés vectoriels concentré en degrés strictement positifs. Comme cela est une condition ouverte, la proposition en découle.

Nous sommes maintenant en mesure de démontrer la proposition suivante, qui donne une description de l'analytifié du faisceau $M(B)$ des $B$-dg-modules simples.

Proposition 4.11 Il existe un isomorphisme naturel entre $M(B)^{\text {an }}$ est le faisceau associé à $F_{B}$.

Preuve: Notons $f: A f f \longrightarrow$ Ste le foncteur d'analytification et $f_{!}: \operatorname{Pr}($ Aff $) \longrightarrow \operatorname{Pr}($ Ste $)$ le foncteur induit sur les préfaisceaux. Rappelons que $M(B)$ est le faisceau associé à un préfaisceau $M^{\prime}(B)$. Il nous suffit donc de construire un morphisme de préfaisceaux

$$
\phi: f_{!}\left(M^{\prime}(B)\right) \longrightarrow F_{B}
$$

qui induise un isomorphisme sur les faisceaux associés.

Pour construire $\phi$ il nous suffit par adjonction de construire un morphisme

$$
M^{\prime}(B) \longrightarrow f^{*}\left(F_{B}\right) .
$$

Soit $X=\operatorname{Spec} A \in A f f$. Nous disposons d'un foncteur d'analytification

$$
-\otimes_{A} \mathcal{O}_{X^{a n}}: C(A) \longrightarrow C\left(\mathcal{O}_{X^{a n}}\right) .
$$

Ce foncteur est un foncteur de Quillen compatible avec l'enrichissement dans $C(\mathbb{C})$. Il induit donc un foncteur de Quillen à gauche sur les catégories de $B$-dg-modules

$$
-\otimes{ }_{A} \mathcal{O}_{X^{a n}}:(A \otimes B)-\operatorname{Mod} \longrightarrow B-\operatorname{Mod}\left(X^{a n}\right),
$$

et donc un foncteur dérivé

$$
-\otimes_{A}^{\mathbb{L}} \mathcal{O}_{X^{a n}}: D(A \otimes B) \longrightarrow D\left(X^{a n}, B\right) .
$$

Pour un point $x \in X(\mathbb{C})$, correspondant à un morphisme d'algèbres $A \longrightarrow \mathbb{C}$, le diagramme suivant

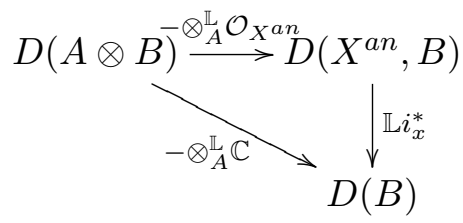

commute à isomorphisme près. Ceci implique que le foncteur $-\otimes_{A}^{\mathbb{L}} \mathcal{O}_{X^{a n}}$ envoie $M^{\prime}(B)(A)$ dans $F_{B}\left(X^{a n}\right)$, et donc induit une application

$$
\phi: M^{\prime}(B)(A) \longrightarrow f^{*}\left(F_{B}\right)(A) .
$$

Cette application est fonctorielle en $A$ et donc fournit un morphisme de préfaisceaux

$$
\phi: M^{\prime}(B) \longrightarrow f^{*}\left(F_{B}\right)
$$


et par adjonction un morphisme de préfaisceaux sur Ste

$$
\phi: f_{!}\left(M^{\prime}(B)\right) \longrightarrow F_{B} .
$$

Il nous reste à voir que ce morphisme induit un isomorphisme fibres à fibres. Pour cela, soit $X \in$ Ste, $x \in X$, et considérons le morphisme induit

$$
f_{!}\left(M^{\prime}(B)\right)\left(X_{x}\right) \longrightarrow F_{B}\left(X_{x}\right) .
$$

D'après la proposition 2.15 ce morphisme est isomorphe à

$$
\operatorname{Colim}_{A \subset \mathcal{O}_{x}} M^{\prime}(B)(A) \longrightarrow \operatorname{Colim}_{x \in U \subset X} F_{B}(U) .
$$

Le corollaire 4.5 permet de calculer la colimite de gauche. En effet, elle implique que l'on a

$$
\operatorname{Colim}_{A \subset \mathcal{O}_{x}} M^{\prime}(B)(A) \simeq M^{\prime}(B)\left(\mathcal{O}_{x}\right) .
$$

Il nous reste donc à montrer que le morphisme naturel

$$
M^{\prime}(B)\left(\mathcal{O}_{x}\right) \longrightarrow F_{B}\left(X_{x}\right)
$$

est une bijection. Nous allons commencer par décrire ce morphisme. Pour cela, soit $x \in U \subset X$ un voisinage ouvert de Stein de $x$ dans $X$. Soit $L(U):=\operatorname{Int}\left(C\left(\mathcal{O}_{U}\right)\right)$ la dg-catégorie des objets fibrants et cofibrants dans $C\left(\mathcal{O}_{U}\right)$ (voir [To1] pour plus de détails sur la construction Int), et $L_{\text {parf }}(U)$ la sous-dg-catégorie pleine de $L(U)$ formée des complexes parfaits de $\mathcal{O}_{U}$-modules. D'après [To1, Thm. 4.2] nous avons une bijection naturelle entre les classes d'isomorphismes d'objets dans $D(U, B)$ et l'ensemble des morphismes $[B, L(U)]$ dans la catégorie homotopique des dg-catégories. Ainsi, $F_{B}(U)$ s'identifie naturellement au sous-ensemble de $\left[B, L_{p a r f}(U)\right]$ formé des morphismes $B \longrightarrow L_{\text {parf }}(U)$ tels que pour tout point $s \in S$, le $B$-dg-module correspondant au morphisme

$$
B \longrightarrow L_{\text {parf }}(U) \stackrel{\mathbb{L} i_{s}^{*}}{\longrightarrow} L_{\text {parf }}(\{s\})
$$

soit un objet simple dans $D(B)$. Cela implique donc que $F_{B}\left(X_{x}\right)$ s'identifie naturellement à un sous-ensemble de $\operatorname{Colim}_{x \in U \subset X}\left[B, L_{\text {parf }}(U)\right]$. Or, $B$ étant propre et lisse, c'est une dgcatégorie homotopiquement de présentation finie (voir [To-Va, Cor. 2.13]), et donc nous avons une bijection

$$
\operatorname{Colim}_{x \in U \subset X}\left[B, L_{\text {parf }}(U)\right] \simeq\left[B, \operatorname{Colim}_{x \in U \subset X} L_{\text {parf }}(U)\right] .
$$

Considérons maintenant $U$ un voisinage ouvert de Stein de $x$ et le foncteur fibre en $x$

$$
C\left(\mathcal{O}_{U}\right) \longrightarrow C\left(\mathcal{O}_{x}\right)
$$

Ce foncteur est de Quillen à gauche, et comme tous les objets dans $C\left(\mathcal{O}_{x}\right)$ sont fibrants il induit un morphisme de dg-catégories

$$
\operatorname{Int}\left(C\left(\mathcal{O}_{U}\right)\right) \longrightarrow \operatorname{Int}\left(C\left(\mathcal{O}_{x}\right)\right)
$$

En se retreignant aux objets parfaits on trouve un morphisme

$$
L_{\text {parf }}(U) \longrightarrow L_{\text {parf }}\left(\mathcal{O}_{x}\right)
$$


des complexes parfaits de $\mathcal{O}_{U}$-modules vers les complexes parfaits sur l'anneau $\mathcal{O}_{x}$. Ce morphisme induit un morphisme de dg-catégories

$$
\operatorname{Colim}_{x \in U \subset X} L_{\text {parf }}(U) \longrightarrow L_{\text {parf }}\left(\mathcal{O}_{x}\right)
$$

qui est une quasi-équivalence. Ainsi, d'après ce que l'on a vu, $F_{B}\left(X_{x}\right)$ s'identifie donc à un sous-ensemble de

$$
\left[B, \operatorname{Colim}_{x \in U \subset X} L_{\text {parf }}(U)\right] \simeq\left[B, L_{\text {parf }}\left(\mathcal{O}_{x}\right)\right] .
$$

D'après la proposition 4.10 il n'est pas difficile de voir que ce sous-ensemble est précisément le sous-ensemble des classes d'isomorphismes de $D\left(B \otimes \mathcal{O}_{x}\right)$ formé des objets compacts $E$ qui sont tels que $E \otimes_{\mathcal{O}_{x}}^{\mathbb{L}} \mathcal{O}_{x} / m_{x}$ soit un objet simple dans $D(B)$. D'après la proposition 4.6 ce sous-ensemble correspond aussi au sous-ensemble $M^{\prime}(B)\left(\mathcal{O}_{x}\right)$ de $\left[B, L_{p a r f}\left(\mathcal{O}_{x}\right)\right]$. Ceci termine la preuve que l'application

$$
M^{\prime}(B)\left(\mathcal{O}_{x}\right) \longrightarrow F_{B}\left(X_{x}\right)
$$

est bijective, et donc termine la preuve de la proposition.

\section{Le théorème de caractérisation}

Nous arrivons maintenant à l'énoncé du théorème principal de ce travail. Pour cela, rappelons qu'une dg-catégorie $T$ est saturée s'il existe une dg-algèbre propre et lisse $B$ et une quasiéquivalence $T \simeq L_{\text {parf }}(B)$ entre $T$ est la dg-catégorie des $B$-dg-modules cofibrants et parfaits (voir [To-Va] pour plus de détails sur cette notion, où cette dg-catégorie est noté $\widehat{B}^{o p}{ }_{p e}$ ).

Théorème 5.1 Soit $X$ un espace analytique connexe, compact et lisse. Alors $X$ est algébrisable si et seulement si la dg-catégorie $L_{\text {parf }}(X)$ est saturée.

La nécessité est bien connue. Lorsque $X$ est un schéma propre et lisse cela est démontré dans [To-Va, Lem. 3.27] (noter que l'on a $L_{\text {parf }}(X) \simeq L_{\text {parf }}\left(X^{a n}\right)$ d'apès GAGA). Dans le cas général où $X$ est un espace algébrique propre et lisse on peut utiliser le lemme de Chow pour montrer que $L_{\text {parf }}(X)$ est saturée (voir l'appendice B pour les détails).

Le reste de cette section est consacré à la preuve de la suffisance de l'énoncé du théorème. Pour cela on se fixe $X$ un espace analytique compact et lisse tel que $L_{p a r f}(X)$ soit saturée. On se fixe aussi une quasi-équivalence $L_{\text {parf }}(B) \simeq L_{\text {parf }}(X)$ avec $B$ une dg-algèbre propre et lisse. Cela revient à se fixer un objet $E \in D_{\text {parf }}(X)$, dont l'enveloppe triangulée et épaisse est $D_{\text {parf }}(X)$, et tel que $\mathbb{R} \underline{E n d}(E)$ soit propre et lisse. Nous nous fixerons plus précisémment un objet fibrant et cofibrant $E \in C\left(\mathcal{O}_{X}\right)$ dans la catégorie de modèles des complexes de $\mathcal{O}_{X}$-modules qui soit un représentant pour $E$. Nous prendrons alors $B:=\underline{E n d}(E)$ la dg-algèbre des endomorphismes de l'objet $E$.

\subsection{Algébrisation de $M(X)$}

Nous allons définir un faisceau $M(X)$, associé à un préfaisceau $M^{\prime}(X)$. 
Définition 5.2 Soit $S \in$ Ste. Nous dirons que $E \in D_{\text {parf }}(X \times S)$ est simple s'il vérifie les deux conditions suivantes:

1. pour tout $s \in S$, le morphisme naturel

$$
\mathbb{C} \longrightarrow \operatorname{Ext}^{0}\left(\mathbb{L} j_{s}^{*}(E), \mathbb{L} j_{s}^{*}(E)\right)
$$

est un isomorphisme,

2. pour tout $s \in S$ et tout $i<0$, on a

$$
\operatorname{Ext}^{i}\left(\mathbb{L} j_{s}^{*}(E), \mathbb{L} j_{s}^{*}(E)\right)=0,
$$

où $j_{s}: X \times\{s\} \hookrightarrow X \times S$ est l'inclusion naturelle et les groupes Ext ${ }^{i}$ sont calculés dans $D(X)$.

On définit alors $M^{\prime}(X)(S)$ comme étant l'ensemble des classes d'isomorphismes d'objets simples dans $D_{\text {parf }}(X \times S)$. Pour un morphisme $f: S \longrightarrow S^{\prime}$ le changement de bases

$$
\mathbb{L} f^{*}: D_{\text {parf }}\left(X \times S^{\prime}\right) \longrightarrow D_{\text {parf }}(X \times S)
$$

préserve les deux propriétés précédentes et induit donc une application

$$
\mathbb{L} f^{*}: M^{\prime}(X)\left(S^{\prime}\right) \longrightarrow M^{\prime}(X)(S) .
$$

Ceci définit un préfaisceau $M^{\prime}(X)$ sur Ste.

Définition 5.3 Le faisceau des complexes parfaits simples sur $X$ est le faisceau associé au préfaisceau $M^{\prime}(X)$. Il sera noté $M(X)$.

Le but de cette partie est de démontrer la proposition suivante.

Proposition 5.4 Le faisceau $M(X)$ est un espace analytique algébrisable.

Preuve: Nous allons montrer plus précisemment qu'il existe un isomorphisme de faisceaux

$$
M(X) \simeq M(B)^{a n} .
$$

Comme nous savons que $M(B)$ est un espace algébrique (voir 4.8) cela impliquera la proposition.

Pour cela nous étudierons la situation générale suivante. Soit $\mathcal{C}$ une catégorie de modèles symétrique monoïdale qui est stable et qui vérifie les conditions de [S-S]. Soit $M$ une $\mathcal{C}$-catégorie de modèles, dont nous noterons $\underline{H o m}$ les morphismes enrichis dans $\mathcal{C}$. Soit $E_{0} \in M$ un objet

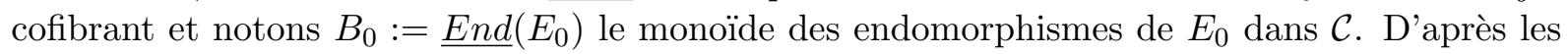
résultats de [S-S], il existe sur $B_{0}-M o d$, la catégorie des $B_{0}$-modules dans $\mathcal{C}$, une structure de catégorie de modèles pour laquelle les fibrations et les équivalences sont définies dans $\mathcal{C}$. On définit alors un foncteur

$$
\phi_{E_{0}}: M \longrightarrow B_{0}-M o d
$$


qui associe à un objet $X \in M$ l'objet $\underline{\operatorname{Hom}}\left(E_{0}, X\right)$ muni de l'action naturelle à gauche du monoïde $\underline{E n d}\left(E_{0}\right)$. Ce foncteur est un foncteur de Quillen à droite, dont l'adjoint à gauche sera noté $\psi_{E_{0}}$. Nous en déduisons une adjonction

$$
\mathbb{L} \psi_{E_{0}}: H o\left(B_{0}-M o d\right) \rightleftarrows H o(M): \mathbb{R} \phi_{E_{0}} .
$$

Soit maintenant $S \in$ Ste un espace de Stein. Nous appliquons les considérations précédentes à la $C\left(\mathcal{O}_{S}\right)$-catégorie de modèles $C\left(\mathcal{O}_{X \times S}\right)$, et à $E_{0}:=E \otimes \mathcal{O}_{S}$ (rappelons que $E \in C\left(\mathcal{O}_{X}\right)$ est un représentant fibrant et cofibrant d'un générateur de $\left.L_{\text {parf }}(X)\right)$. Nous avons donc un foncteur

$$
\phi_{E_{0}}: D(X \times S) \longrightarrow D\left(B_{0}-M o d\right),
$$

où $B_{0}=\underline{E n d}\left(E_{0}\right)$. On dispose de plus d'un morphisme naturel de monoïdes dans $C\left(\mathcal{O}_{S}\right)$

$$
B \otimes \mathcal{O}_{S}=\underline{\operatorname{End}}(E) \otimes \mathcal{O}_{S} \longrightarrow \underline{\operatorname{End}}\left(E_{0}\right)
$$

induit par le foncteur image inverse

$$
C(\mathbb{C}) \longrightarrow C\left(\mathcal{O}_{S}\right)
$$

associé au morphisme $S \longrightarrow *$. Ce morphisme induit un foncteur d'oubli sur les catégories de modules

$$
D\left(B_{0}-M o d\right) \longrightarrow D(B-M o d)=D(S, B) .
$$

En composant avec $\phi_{E_{0}}$ on obtient un foncteur

$$
\phi: D(X \times S) \longrightarrow D(S, B) .
$$

Ce foncteur est le composé de deux foncteurs adjoints à droite, et donc possède un adjoint à gauche

$$
\psi: D(S, B) \longrightarrow D\left(B_{0}-M o d\right) .
$$

Pour $F \in D(X \times S)$, l'objet de $D(S)$ sous-jacent à $\phi(F)$ est $\mathbb{R} p_{*}\left(E_{0}^{\vee} \otimes^{\mathbb{L}} F\right)$, où $E_{0}^{\vee}$ est le dual du complexe parfait $E_{0}$ et $p: X \times S \longrightarrow S$ est la projection sur le second facteur. Comme $E_{0}^{\vee}$ est parfait sur $X \times S$, la proposition 3.2 implique que le foncteur $\phi$ envoie $D_{\text {parf }}(X \times S)$ dans la sous-catégorie pleine de $D(S, B)$ formée des objets qui sont parfaits comme complexes de $\mathcal{O}_{S}$-modules.

Nous avons donc un foncteur induit entre catégories d'objets parfaits

$$
\phi: D_{\text {parf }}(X \times S) \longrightarrow D_{\text {parf }}(S, B) .
$$

La propriété de changement de bases (voir corollaire 3.6) implique que $\phi$ est compatible, à isomorphisme près, aux changement de bases induit par des morphismes $S^{\prime} \longrightarrow S$. De plus, comme $E$ est générateur de $L_{\text {parf }}(X)$, le foncteur

$$
\phi: D_{\text {parf }}(X \times S) \longrightarrow D_{\text {parf }}(S, B)
$$

est une équivalence lorsque $S=*$ est réduit à un point. Ces deux propriétés impliquent que le foncteur

$$
\phi: D_{\text {parf }}(X \times S) \longrightarrow D_{\text {parf }}(S, B)
$$


préserve aussi les objets simples au sens des définitions 4.9 et 5.2. Il induit donc un morphisme de faisceaux sur Ste

$$
\phi: M(X) \longrightarrow M(B)^{a n}
$$

Il nous reste à montrer que $\phi$ est un isomorphisme. Pour cela, nous allons montrer que pour tout $S \in$ Ste, le foncteur

$$
\phi: D_{\text {parf }}(X \times S) \longrightarrow D_{\text {parf }}(S, B)
$$

est une équivalence. On considère le foncteur

$$
\psi: D(S, B) \longrightarrow D(X \times S),
$$

adjoint à gauche du foncteur $\phi: D(X \times S) \longrightarrow D(S, B)$.

Lemme 5.5 Le foncteur

$$
\psi: D(S, B) \longrightarrow D(X \times S)
$$

préserve les objets parfaits.

Preuve du lemme: Comme nous l'avons vu lors de la preuve de la proposition 4.10, $D_{\text {parf }}(S, B)$ est la plus petite sous-catégorie triangulée épaisse de $D(S, B)$ contenant les objets de la forme $B \otimes F$, où $F \in D_{\text {parf }}(S)$ (avec la structure de $B$-dg-module est donnée par l'action de $B$ sur ellemême). Ainsi, pour montrer que $\psi$ préserve les objets parfaits il suffit de montrer que $\psi(B \otimes F)$ est parfait sur $X \times S$ lorsque $F$ est parfait sur $S$. Or, on voit par adjonction que l'on a

$$
\psi(B \otimes F) \simeq E \otimes^{\mathbb{L}} F .
$$

D'après le lemme on dispose d'une adjonction

$$
\psi: D_{\text {parf }}(S, B) \rightleftarrows D_{\text {parf }}(X \times S): \phi .
$$

De plus, cette adjonction est compatible aux changements de bases sur $S$. Ainsi, pour voir que les morphismes d'adjonction

$$
I d \longrightarrow \phi \psi \quad \psi \phi \longrightarrow I d
$$

sont des isomorphismes on peut supposer que $S$ est égal à un point (cela est justifiable du fait que tous les objets considérés sont des complexes parfaits sur $S$ et sur $X \times S$, et donc qu'être un quasi-isomorphisme peut se tester points par points). Mais dans ce cas, le fait que cette adjonction soit une équivalence découle du fait que $E$ est un générateur de la dg-catégorie $L_{\text {parf }}(X)$.

Nous venons de voir que

$$
\phi: D_{\text {parf }}(X \times S) \longrightarrow D_{\text {parf }}(S, B)
$$

était une équivalence compatible aux changements de bases en $S$. Cela implique qu'en passant aux classes d'isomorphismes d'objets simples ce foncteur induit un isomorphisme de faisceaux

$$
\phi: M(X) \simeq M(B)^{a n} .
$$




\subsection{Plongement de $X$ dans $M(X)$}

Le but de cette section est de définir un plongement ouvert de $X$ dans l'espace algébrisable $M(X) \simeq M(B)^{a n}$. Pour cela on envoie un point $x$ de $X$ dans le faisceau gratte-ciel $k(x) \in$ $D_{\text {parf }}(X)$, qui est un objet simple et donc un point de $M(X)$.

Proposition 5.6 Il existe un morphisme injectif et étale

$$
j: X \longrightarrow M(X) .
$$

Preuve: Soit $S \in$ Ste un espace de Stein et $u: S \longrightarrow X$ un morphisme, considéré comme un élément de $X(S)$. Notons $\Gamma(u) \subset X \times S$ le graphe de $u$, qui est un sous-ensemble analytique de $X \times S$ dont nous noterons $I(u) \subset \mathcal{O}_{X \times S}$ l'idéal. Notons $F(u)$ le faisceau cohérent $\mathcal{O}_{X \times S} / I(u)$, que l'on considère comme un objet dans $D_{\text {parf }}(X \times S)$. L'objet $F(u)$ est simple au sens de la définition 5.2 , car pour $s \in S$ on a

$$
\mathbb{R} j_{s}^{*}(F(u)) \simeq k(u(s)),
$$

où $k(u(s))$ est le faisceau gratte-ciel centré au point $u(s) \in X$. La construction $u \mapsto F(u)$ induit ainsi une application

$$
j: X(S) \longrightarrow M^{\prime}(X)(S) .
$$

Lorsque $S$ varie dans $S t e$ cela définit un morphisme de préfaisceaux

$$
j: X \longrightarrow M^{\prime}(X)
$$

et donc de faisceaux

$$
j: X \longrightarrow M(X) .
$$

La morphisme $j$ est injectif car si deux points $x$ et $x^{\prime}$ dans $X$ sont tels que $k(x)$ et $k\left(x^{\prime}\right)$ soient isomorphes dans $D(X)$ alors $x=x^{\prime}$ pour des raisons de supports. Il nous reste à montrer que $j$ est étale.

Nous commencerons par montrer que $j$ est un monomorphisme. Pour cela, soit $u, v: S \longrightarrow X$ deux morphismes avec $S \in$ Ste tels que $j(u)=j(v)$. Dire que $j(u)=j(v)$ dans $M(X)(S)$ est équivalent à dire que les faisceaux structuraux des graphes $\Gamma(u)$ et $\Gamma(v)$ de $u$ et $v$ sont isomorphes comme faisceaux cohérents sur $X \times S$, au moins localement sur $S$. Comme la propriété $u=v$ est locale sur $S$, on peut supposer que faisceaux structuraux des graphes $\Gamma(u)$ et $\Gamma(v)$ sont isomorphes. Cela implique facilement que les sous-espaces analytiques définis par ces graphes sont égaux (en tant que sous-espaces analytiques de $X \times S$ ). Ceci implique bien entendu que les morphismes $u$ et $v$ sont égaux. Ainsi, $j$ est non-seulement injectif mais aussi un monomorphisme. Il nous reste donc à montrer que $j$ est aussi formellement lisse, ce qui impliquera qu'il est étale.

Soit donc $S \in$ Ste un espace de Stein et $i: S_{0} \subset S$ un sous-espace analytique fermé défini par un idéal de carré nul. Supposons que l'on ait un diagramme commutatif de faisceaux

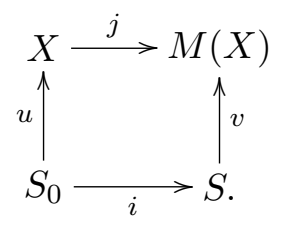


On cherche à montrer qu'il existe un morphisme $w: S \longrightarrow X$ faisant commuter le diagramme

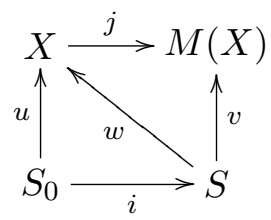

Comme nous savons déjà que $j$ est un monomorphisme, et donc non-ramifié, l'existence de ce morphisme $w$ est une condition locale sur $S$. On peut donc supposer que le morphisme $v: S \longrightarrow M(X)$ se factorise par un morphisme $v^{\prime}: S \longrightarrow M^{\prime}(X)$ de sorte à ce que le diagramme

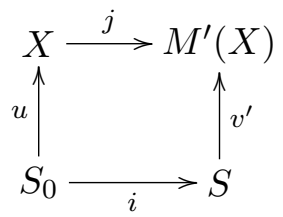

soit un diagramme commutatif de préfaisceaux. La donnée de ce dernier diagramme est équivalente à la donnée d'un couple $(u, E)$, où

1. $u: S_{0} \longrightarrow X$ est un morphisme dont le faisceau structural du graphe $\Gamma(u) \subset X \times S_{0}$ sera noté $E_{0}(u)$.

2. $E \in D_{\text {parf }}(X \times S)$ est un objet simple tel que $\mathbb{L} i^{*}(E)$ et $E_{0}(u)$ soit isomorphes dans $D_{\text {parf }}\left(X \times S_{0}\right)$.

Pour montrer que le relèvement $w$ comme ci-dessus existe il suffit de montrer qu'il existe un morphisme $w^{\prime}: S \longrightarrow X$, dont le faisceau structural du graphe sera noté $E\left(w^{\prime}\right) \in D_{\text {parf }}(X \times S)$, et qui est tel que

$$
E\left(w^{\prime}\right) \simeq E \quad w^{\prime} \circ i=u .
$$

Comme le morphisme $j$ est un monomorphisme il nous suffit en réalité de montrer l'existence de $w^{\prime}$ tel que $E\left(w^{\prime}\right) \simeq E$, la seconde condition étant alors automatique.

Pour commencer, nous avons $E \in D_{\text {parf }}(X \times S)$ tel que $\mathbb{L} i^{*}(E)$ soit un faisceau cohérent et plat sur $S_{0}$. Comme dans [An-To, Lem. 5.2] cela implique que $E$ est lui-même un faisceau cohérent et plat sur $S$. On considère alors la projection $p: X \times S \longrightarrow S$, et l'image directe $\mathbb{R} p_{*}(E)$ de $E$ sur $S$. Par la propriété de changement de bases du corollaire 3.6 on voit que pour tout $s \in S$, le complexe $\mathbb{L} j_{s}^{*} \mathbb{R} p_{*}(E)$ est quasi-isomorphe à $\mathbb{C}$ concentré en degré zéro. Cela implique que $\mathbb{R} p_{*}(E)$ est un fibré en droite $L$ sur $S$. Quitte à restreindre $S$ si nécessaire on peut aussi supposer que $L$ est trivial et choisir une trivialisation $e: \mathcal{O}_{S} \simeq \mathbb{R} p_{*}(E)$. Le morphisme $e$ correspond par adjonction à un morphisme de faisceaux cohérents $f: \mathcal{O}_{X \times S} \longrightarrow E$. En utilisant que $\mathbb{L} i^{*}(E) \simeq E_{0}(u)$ on voit, points par points sur $X \times S$, que le morphisme $f$ est un épimorphisme. Ainsi, $E$ est-il isomorphe au faisceau structural d'un sous-espace analytique $Z \subset X \times S$, plat sur $S$. Enfin, le morphisme $Z \longrightarrow S$ est plat et tel que le morphisme induit

$$
Z \times{ }_{S} S_{0} \simeq \Gamma(u) \longrightarrow S_{0}
$$

soit un isomorphisme, et donc est lui-même un isomorphisme. Cela implique l'existence d'un morphisme $w^{\prime}: S \longrightarrow X$ dont le graphe est égal au sous-espace $Z$. On a donc bien $E\left(w^{\prime}\right) \simeq$ $\mathcal{O}_{Z} \simeq E$. 


\subsection{Algébrisation de $X$}

Pour achever la preuve du théorème il nous reste à démontrer la proprosition suivante.

Proposition 5.7 Soit $M$ un espace algébrique quasi-séparé (i.e. le morphisme diagonal $M \longrightarrow$ $M \times M$ est quasi-compact). S'il existe un morphisme injectif et étale

$$
j: X \longrightarrow M^{a n}
$$

alors $X$ est algébrisable (i.e. il existe un espace algébrique $Y$ tel que $Y^{a n} \simeq X$ ).

Preuve: Soit $\left\{U_{i} \longrightarrow M\right\}$ une famille couvrante de morphismes étales avec $U_{i}$ des schémas affines. Comme $M$ est quasi-séparé chacun des morphismes $U_{i} \longrightarrow M$ est étale et de type fini. L'image de $U_{i} \longrightarrow M$ est donc un sous-espace algébrique ouvert $M_{i} \subset M$ de type fini. Ainsi, l'espace algébrique $M$ est une réunion de sous-espaces ouverts de type fini. Par compacité de $X$ on peut donc supposer que $j$ se factorise en $X \longrightarrow\left(M^{\prime}\right)^{a n} \subset M^{a n}$, avec $M^{\prime}$ un sous-espace algébrique ouvert de type fini. En d'autres termes on peut supposer que $M$ est de type fini.

L'espace analytique $X$ est irréductible (au sens analytique), et donc le morphisme $j: X \longrightarrow$ $M^{a n}$ se factorise en $X \longrightarrow Z \subset M^{a n}$, où $Z$ est une composante irréductible analytique de $M^{a n}$. Comme le morphisme composé

$$
X \longrightarrow Z \hookrightarrow M^{a n}
$$

est étale et que $Z \longrightarrow M^{a n}$ est un monomorphisme, on voit que le morphisme $X \longrightarrow Z$ est encore injectif et étale. De plus, d'après [SGA1, Exp. XII, Prop. 2.4], il existe une composante irréductible $M_{0}$ de $M$ (au sens algébrique) tel que $Z \simeq M_{0}^{a n}$. Cela implique que l'on peut supposer que $M$ est irréductible. De la même façon, comme $X$ est réduit on pourra supposer que $M$ est réduit.

L'immersion ouverte $j: X \hookrightarrow M^{a n}$ induit un morphisme injectif sur les corps de fonctions méromorphes

$$
K\left(M^{a n}\right) \hookrightarrow K(X) .
$$

De plus, comme $j$ est une immersion ouverte nous avons les égalités

$$
\operatorname{dim}(X)=\operatorname{dim}\left(M^{a n}\right)=\operatorname{dim}(M)
$$

(voir [SGA1, Exp. XII, Prop. 2.1]). Comme $M$ est quasi-séparé il existe un ouvert affine dense $U$ de $M$ ([Kn, Prop. I.5.19]) qui est de dimension $n=\operatorname{dim}(X)$. Ainsi, on trouve un plongement

$$
K(U) \simeq K(M) \subset K\left(M^{a n}\right) \hookrightarrow K(X),
$$

où $K(U)$ et $K(M)$ désigne les corps de fractions rationnelles sur $U$ et $M$. Comme $K(U)$ est de degré de transcendance égal à $n$, l'espace analytique $X$ est un espace de Moishezon, et donc est algébrisable d'après $[\mathrm{Ar}]$.

Pour finir la preuve du théorème 5.1 nous appliquons la proposition 5.7 au morphisme injectif et étale

$$
j: X \longrightarrow M(X) \simeq M(B)^{a n}
$$

de la proposition 5.6. Ceci est possible car $M(B)$ est un espace algébrique quasi-séparé d'après le théorème 4.8 . 


\section{A Dg-catégories saturées et catégories triangulées saturées}

Dans cette section nous comparons les notions de saturation dans les cadres des dg-catégories et des catégories triangulées, et nous comparons ainsi les notions de [B-V] et [To-Va]. Pour cela nous nous restreindrons au cas où $k$ est un corps.

Commençons par rappeler la définition d'une catégorie triangulée $k$-linéaire saturée au sens de [B-V, Def. 1.2].

Définition A.1 Une catégorie triangulée $k$-linéaire $\mathcal{D}$ est saturée si elle vérifie les deux conditions suivantes.

1. Pour toute paire d'objets $(x, y)$ dans $\mathcal{D}$, on a

$$
\operatorname{Dim}_{k} \operatorname{Ext}^{*}(x, y)<\infty,
$$

où $\operatorname{Ext}^{*}(x, y)=\oplus_{i \in \mathbb{Z}}[x, y[i]]$.

2. Tout foncteur cohomologique

$$
H: \mathcal{D}^{o p} \longrightarrow V e c t(k)
$$

tel que pour tout $x \in \mathcal{D}$ on ait

$$
\operatorname{Dim}_{k} \oplus_{i \in \mathbb{Z}} H(x[i])<\infty,
$$

est représentable.

Comme on peut s'y attendre la notion de saturation pour les dg-catégories est plus forte que celle pour les catégories triangulées.

Proposition A.2 Soit $T$ une dg-catégorie saturée sur $k$. Alors la catégorie $[T]$, munie de sa structure triangulée naturelle (voir [To-Va, §2.2]), est saturée au sens de la définition A.1.

Preuve: Par définition on peut supposer que $T$ est de la forme $L_{\text {parf }}(B)$, pour $B$ une dgalgèbre propre et lisse sur $k$. Dans ce cas $[T] \simeq D_{\text {parf }}(B)$ est la catégorie triangulée des $B$ dg-modules parfaits (munie de sa structure triangulée naturelle). On dispose d'un foncteur bi-exact

$$
D_{\text {parf }}\left(B \otimes B^{o p}\right) \times D_{\text {parf }}(B) \longrightarrow D_{\text {parf }}(B),
$$

qui envoie un $B \otimes B^{o p}$-dg-module $P$ et un $B$-dg-module $M$ sur $P \otimes \mathbb{L}_{B} M$. Ce foncteur envoie $(B, M)$ sur $M$, et $\left(B \otimes B^{o p}, M\right)$ sur le $B$-dg-module libre $B \otimes M$. Comme $B$ appartient à l'enveloppe triangulée épaisse de $B \otimes B^{o p}$, il existe un entier $n$ tel que $B \in<B \otimes B^{o p}>_{n}$ (avec les notations de [B-V]). Cela implique facilement que $M \in<B>_{n}$, et ce pour tout $M$. En d'autres termes, $B$ est un générateur fort (strong generator dans la terminologie de $[\mathrm{B}-\mathrm{V}]$ ) de $D_{\text {parf }}(B)$. Comme $D_{\text {parf }}(B)$ satisfait la condition (1) de la définition A.1, le théorème principal de $[\mathrm{B}-\mathrm{V}]$ implique que $D_{\text {parf }}(B)$ est saturée.

Nous ne savons pas s'il est raisonnable d'attendre à ce qu'une réciproque de la proposition A.2 soit vraie. On dispose cependant de la réciproque partielle suivante. 
Proposition A.3 Soit $T$ une dg-catégorie triangulée (voir [To-Va, Def. 2.4]). On suppose que les trois conditions suivantes sont satisfaites.

1. La catégorie triangulée $[T] \simeq D_{\text {parf }}\left(T^{o p}\right)$ possède un générateur classique (au sens de $[B-V])$.

2. Pour toute paire d'objets $(x, y)$ dans $[T]$, on a

$$
\operatorname{Dim}_{k} \operatorname{Ext}^{*}(x, y)<\infty
$$

où $\operatorname{Ext}^{*}(x, y)=\oplus_{i \in \mathbb{Z}}[x, y[i]]$.

3. La catégorie triangulée $\left[\widehat{T \otimes T^{\circ}}{ }_{p e}\right]$ est saturée.

Alors la dg-catégorie T est saturée.

Preuve: La première hypothèse implique qu'il existe une dg-algèbre $B$ telle que $T$ soit quasiéquivalente à $L_{\text {parf }}(B)$. La seconde hypothèse implique de plus que $B$ est une dg-algèbre propre.

La dg-catégorie $\widehat{T \otimes T^{o p}}{ }_{p e}$ est quasi-équivalente à $L_{p a r f}\left(B \otimes B^{o p}\right)$. On considère alors le foncteur cohomologique

$$
H: D_{\text {parf }}\left(B \otimes B^{o p}\right) \longrightarrow V e c t(k),
$$

qui à $M$ un $B \otimes B^{o p}$ dg-module parfait associe $[M, B]$, l'ensemble des morphismes de $B$ vers $M$ pris dans $D\left(B \otimes B^{o p}\right)$ la catégorie dérivée de tous les $B \otimes B^{o p}$-dg-modules. Ce foncteur vérifie bien la condition de finitude (2) de la définition A.1 car $B \otimes B^{o p}$ est un générateur classique de $D_{\text {parf }}\left(B \otimes B^{o p}\right)$, et $H\left(B \otimes B^{o p}[n]\right)=H^{-n}(B)$ pour tout $n \in \mathbb{Z}$. Il existe donc un $B \otimes B^{o p}$ dg-module $P$ parfait qui représente le foncteur $H$. Choisissons un isomorphisme de foncteurs $H \simeq[-, P]$. L'identité de $P$ définit alors un morphisme dans $D\left(B \otimes B^{o p}\right)$

$$
u: P \longrightarrow B
$$

Par construction, ce morphisme est tel que pour tout $M \in D_{\text {parf }}\left(B \otimes B^{o p}\right)$ le morphisme induit

$$
u_{*}:[M, P] \longrightarrow[M, B]
$$

soit bijectif. En appliquant cela aux $M=B \otimes B^{o p}[n]$, on trouve que pour tout $n \in \mathbb{Z}$ le morphisme induit

$$
H^{n}(u): H^{n}(P) \longrightarrow H^{n}(B)
$$

est un isomorphisme. Ainsi, $u$ est un isomorphisme dans $D\left(B \otimes B^{o p}\right)$ ce qui implique en particulier que $B$ est un objet de $D_{\text {parf }}\left(B \otimes B^{o p}\right)$. Par définition cela signifie que $B$ est lisse. Ainsi, $T \simeq L_{\text {parf }}(B)$ avec $B$ propre et lisse et donc $T$ est saturée au sens de [To-Va].

\section{B Catégories dérivées des espaces algébriques propres et lisses}

Le but de ce second appendice est de montrer la proposition suivante. 
Proposition B.1 Soit $X$ un espace algébrique propre et lisse sur un corps $k$ de caractéristique nulle. Alors la dg-catégorie $L_{\text {parf }}(X)$ est saturée.

Preuve: Lorsque $X$ est un schéma cela est démontré dans [To-Va, Lem. 3.27]. La même preuve marcherait aussi dans le cas général si l'on savait que $D_{\text {parf }}(X)$ possède un générateur, ce que nous allons montrer. Par le lemme de Chow et la résolution des singularités, il existe un schéma propre et lisse $X^{\prime}$ et un morphisme birationnel surjectif $p: X^{\prime} \longrightarrow X$. Ce morphisme vérifie $\mathbb{R} p_{*}\left(\mathcal{O}_{X^{\prime}}\right) \simeq \mathcal{O}_{X}$ car $X$ est lisse et donc à singularités rationnelles. Ceci implique par la formule de projection que le foncteur

$$
\mathbb{L} p^{*}: D_{\text {parf }}(X) \longrightarrow D_{\text {parf }}\left(X^{\prime}\right)
$$

est pleinement fidèle. Mais cela implique en particulier que l'image d'un générateur classique de $D_{\text {parf }}\left(X^{\prime}\right)$ par $\mathbb{R} p_{*}$ est un générateur classique de $D_{\text {parf }}(X)$.

\section{Catégories dérivées non bornées}

Dans cette dernière section nous définissons une catégorie de modèles monoïdale symétrique dont la catégorie homotopique est la catégorie dérivée non-bornée des complexes de $\mathcal{O}$-modules sur un site annelé $(C, \mathcal{O})$. L'existence de cette structure de modèles nous permet alors de considérer les 4 opérations standards (images directes, inverses, produits tensoriels et Hom internes) au niveau des catégories dérivées non bornées.

Fixons $(C, \mathcal{O})$ un petit site annelé en anneaux commutatifs. Nous notons $C(\mathcal{O})$ la catégorie des complexes de préfaisceaux de $\mathcal{O}$-modules sur $C$. Ses objets sont la donnée de $\mathcal{O}(U)$-modules $M(U)$ pour tout objet $U$ de $C$, fonctoriels en $U$ en un sens évident. Nous munissons la catégorie $C(\mathcal{O})$ d'une première structure de modèles, appelée la structure niveaux par niveaux pour laquelle on a:

1. Un morphisme $f: M \longrightarrow N$ est une fibration si pour tout $U \in C$ le morphisme de complexes de $\mathcal{O}(U)$-modules $M(U) \longrightarrow N(U)$ est un épimorphisme.

2. Un morphisme $f: M \longrightarrow N$ est une équivalence si pour tout $U \in C$ le morphisme $M(U) \longrightarrow N(U)$ est un quasi-isomorphisme.

Il est facile de voir que ces notions définissent une structure de catégorie de modèles engendrée par cofibrations, propre et cellulaire sur $C(\mathcal{O})$.

Nous introduisons maintenant la notion suivante d'équivalence locale. Pour cela rappelons que pour $M \in C(\mathcal{O})$ et tout $n \in \mathbb{Z}$ on dispose de préfaisceaux $H_{p r}^{n}(M)$ sur $C$, qui à $U$ associe le $n$-ème groupe de cohomologie du complexe $M(U)$. Le faisceau associé à ce préfaisceau sera noté $H^{n}(M)$.

Définition C.1 Un morphisme $f: M \longrightarrow N$ dans $C(\mathcal{O})$ est une équivalence locale si pour tout $n \in \mathbb{Z}$ le morphisme induit

$$
H^{n}(M) \longrightarrow H^{n}(N)
$$

est un isomorphisme de faisceaux sur $C$. 
La définition précédente permet de définir des nouvelles notions de cofibrations, fibrations et équivalences locales sur $C(\mathcal{O})$. Pour cela nous définissons les cofibrations locales comme étant les cofibrations pour la structure niveaux par niveaux, et les fibrations locales comme étant les morphismes possèdant la propriété de relèvement à droite des cofibrations qui sont aussi des équivalences locales.

Théorème C.2 Les notions précédente de fibrations, cofibrations et équivalences locales définissent une structure de catégorie de modèles sur $C(\mathcal{O})$, propre et engendrée par cofibrations.

Nous ne donnerons pas la preuve de ce théorème, elle est tout à fait similaire à la preuve de l'existence de la structure locale projective pour les préfaisceaux simpliciaux donnée par exemple dans [HAGI, Thm. 3.4.1].

Remarque C.3 La catégorie homotopique $H o(C(\mathcal{O}))$ est naturellement équivalente à la catégorie dérivée des faisceaux de $\mathcal{O}$-modules sur le site $C$. En effet, le foncteur d'inclusion des complexes de faisceaux de $\mathcal{O}$-modules dans les complexes de préfaisceaux de $\mathcal{O}$-modules induit clairement une équivalence après avoir localisé le long des équivalences locales.

La catégorie $C(\mathcal{O})$ est naturellement munie d'une structure monoïdale symétrique, notée $\otimes$, et pour la quelle on a

$$
(M \otimes N)(U)=M(U) \otimes N(U)
$$

Proposition C.4 Supposons que $C$ possède des produits finis, alors $C(\mathcal{O})$ munie de la structure monoïdale $\otimes$ est une catégorie de modèles monoïdale au sens de [Ho, §4]. Elle vérifie de plus l'axiome du monoïde de [S-S].

Esquisse de preuve: On commence par montrer que $C(\mathcal{O})$ est une catégorie de modèles monoïdale lorsqu'elle est munie de sa structure niveaux par niveaux. Pour cela, nous appliquerons l'énoncé général de [Ho, Cor. 4.2.5], et nous aurons donc besoin d'expliciter les ensembles générateurs $I$ et $J$ de cofibrations et cofibrations triviales. Pour tout $U \in C$, le foncteur d'évaluation en $U$

$$
j_{U}^{*}: C(\mathcal{O}) \longrightarrow C(\mathcal{O}(U)),
$$

qui à $M$ associe le complexe de $\mathcal{O}(U)$-modules $M(U)$, est de Quillen à droite (pour la structure projective sur $C(\mathcal{O}(U))$ de [Ho], pour la quelle les fibrations sont les épimorphismes et les équivalences sont les quasi-isomorphismes). L'adjoint à gauche de ce foncteur sera noté

$$
\left(j_{U}\right) !: C(\mathcal{O}(U)) \longrightarrow C(\mathcal{O}) .
$$

Si $I(U)$ et $J(U)$ désigne alors les ensembles générateurs de cofibrations et cofibrations triviales dans $C(\mathcal{O}(U))$, il est facile de voir que

$$
I:=\left\{\left(j_{U}\right) !(u)\right\}_{U \in C, u \in I(U)} \quad J:=\left\{\left(j_{U}\right) !(u)\right\}_{U \in C, u \in J(U)}
$$

sont des ensembles générateurs de cofibrations et cofibrations triviales pour la structure niveaux par niveaux de $C(\mathcal{O})$. 
Soit maintenant

$$
\left(j_{U}\right) !(A) \longrightarrow\left(j_{U}\right)_{!}(B) \quad\left(j_{V}\right) !(C) \longrightarrow\left(j_{V}\right) !(D)
$$

deux éléments de $I$, pour deux objets $U, V \in C$. Alors, il est facile de voir qu'il existe un isomorphisme naturel

$$
\left(j_{U}\right) !(X) \otimes\left(j_{V}\right) !(Y) \simeq\left(j_{U \times V}\right) !(X \otimes Y) .
$$

Ainsi, le morphisme induit

$$
\left(\left(j_{U}\right) !(A) \otimes\left(j_{V}\right) !(D)\right) \coprod_{\left(\left(j_{U}\right) !(A) \otimes\left(j_{V}\right) !(C)\right)}\left(\left(j_{U}\right) !(B) \otimes\left(j_{V}\right) !(C)\right) \longrightarrow\left(j_{U}\right)_{!}(B) \otimes\left(j_{V}\right)_{!}(D)
$$

est-il isomorphe au morphisme naturel

$$
\left(j_{U \times V}\right) !\left((A \otimes D) \coprod_{A \otimes C} B \otimes C\right) \longrightarrow\left(j_{U \times V}\right) !((B \otimes D)) .
$$

Ce dernier morphisme est bien une cofibration car $\left(j_{U \times V}\right)$ ! est de Quillen à gauche. De plus cette cofibration est aussi une équivalence si l'un des deux morphismes $A \longrightarrow B$ ou $C \longrightarrow D$ est une équivalence. Ceci fini de montrer que $C(\mathcal{O})$ munie de sa structure niveaux par niveaux est une catégorie de modèles monoïdale. On en déduit aisément que $C(\mathcal{O})$ munie de sa structure de modèles locale est encore une catégorie de modèles monoïdale.

Il reste à voir que $C(\mathcal{O})$ vérifie à l'axiome du monoïde [S-S]. Pour cela il suffit de montrer que si $X \longrightarrow Y$ est une cofibration triviale locale dans $C(\mathcal{O})$, et $M$ est un objet de $C(\mathcal{O})$ alors le morphisme $X \otimes M \longrightarrow Y \otimes M$ est une équivalence locale. On considère la suite de morphismes dans $C(\mathcal{O})$

$$
X \otimes M \longrightarrow Y \otimes M \longrightarrow Y / X \otimes M .
$$

Comme $Y / X$ est cofibrant dans $C(\mathcal{O})$, il est plat niveaux par niveaux. Ainsi, la suite précédente est une suite exacte, et il nous suffit donc de montrer que $Y / X \otimes M$ est équivalent à 0 (pour la structure locale). Pour cela, soit $p: Q M \longrightarrow M$ un remplacement cofibrant de $M$. Le morphisme $p$ est une fibration triviale et donc une équivalence niveaux par niveaux. Comme $Y / X$ est plat niveaux par niveaux on voit que le morphisme induit

$$
Y / X \otimes Q M \longrightarrow Y / X \otimes M
$$

est une équivalence niveaux par niveaux. Ainsi, on a un isomorphisme dans $H o(C(\mathcal{O}))$

$$
Y / X \otimes^{\mathbb{L}} M \simeq Y / X \otimes M,
$$

ce qui montre bien que $Y / X \otimes M$ est équivalent à 0 .

En corollaire de la proposition, on peut définir une catégorie monoïdale symétrique $D(C, \mathcal{O}):=$ $H o(C(\mathcal{O}))$, pour la structure monoïdale dérivée $\otimes^{\mathbb{L}}$. De plus, cette structure monoïdale est fermée. Le fait que l'axiome du monoïde soit vérifié entraine l'existence de structures de modèles sur les catégories de monoïdes et de modules dans $C(\mathcal{O})$ comme démontré dans [S-S]. 
Pour terminer, supposons que $f: C \longrightarrow D$ soit un foncteur exact à gauche et continu entre sites possédant des limites finies. Supposons que $\mathcal{O}_{C}$ et $\mathcal{O}_{D}$ soient deux faisceaux d'anneaux commutatifs sur $C$ et $D$ munis d'un morphisme

$$
\mathcal{O}_{C} \longrightarrow f^{-1}\left(\mathcal{O}_{D}\right)
$$

On peut alors définir une adjonction

$$
f_{!}: C\left(\mathcal{O}_{C}\right) \rightleftarrows C\left(\mathcal{O}_{D}\right): f^{*}
$$

dont il est facile de montrer qu'il s'agit d'une adjonction de Quillen en remarquant que $f$ ! commute à la formation des préfaisceaux de cohomologie $H_{p r}^{*}$.

\section{Références}

[An-To] M. Anel, B. Toën, Dénombrabilité des classes d'équivalences dérivées de variétés algébriques, Preprint math.AG/0611545.

[Ar] M. Artin, Algebraization of formal moduli II. Existence of modifications, Ann. of Math. (2) 911970 88-135.

[Ba-St] C. Banica, O. Stanasila, Algebraic methods in the global theory of complex spaces. Translated from the Romanian. Editura Academiei, Bucharest; John Wiley \& Sons, London-New York-Sydney, 1976. 296 pp.

[B-V] A. Bondal, M. Van Den Bergh, Generators and representability of functors in commutative and non-commutative geometry, Mosc. Math. J. 3 (2003), no. 1, 1-36.

[Du] D. Dugger, Universal Homotopy Theories, Adv. in Math. 164 (2001), 144-176.

[D-H-I] D. Dugger, S. Hollander, D. Isaksen, Hypercovers and simplicial presheaves, Math. Proc. Cambridge Philos. Soc. 136 (2004), 9-51.

[Gr-Re] H. Grauert, R.Remmert, Coherent analytic sheaves, Grundlehren der Mathematischen Wissenschaften 265, Springer-Verlag, Berlin, 1984. xviii+249 pp.

[Hi] P. Hirschhorn, Model categories and their localizations, Math. Surveys and Monographs Series 99, AMS, Providence, 2003.

[H-Si] A. Hirschowitz, C. Simpson, Descente pour les n-champs, Preprint math.AG/9807049.

[Ho] M. Hovey, Model categories, Mathematical surveys and monographs, Vol. 63, Amer. Math. Soc., Providence 1998.

[Kn] D. Knutson, Algebraic Spaces, Lecture Notes in Mathematics 203, Springer-Verlag 1971.

[Ko-So] M. Kontsevich, Y. Soibelmann, Notes on A-infinity algebras, A-infinity categories and non-commutative geometry. I, arXiv Preprint math.RA/0606241. 
[L] M. Lieblich, Moduli of complexes on a proper morphism, J. Algebraic Geom. 15 (2006), no. $1,175-206$.

[SGA1] Revêtements étales et groupe fondamental, [Séminaire de géométrie algébrique du Bois Marie 1960-61, dirigé par A. Grothendieck, Documents Mathématiques (Paris) 3, Société Mathématique de France, Paris, 2003. xviii+327 pp.

[S-S] S. Schwede, B. Shipley, Algebras and modules in monoidal model categories, Proc. London Math. Soc. (3) 80 (2000), 491-511.

[Si] C. Simpson, Algebraic (geometric) n-stacks, Preprint math.AG/9609014.

[To1] B. Toën, The homotopy theory of dg-caregories and derived Morita theory, à paraître dans Invent. Math., pré-publication math.AG/0408337.

[To2] B. Toën, Champs algébriques: cours 2, cours de Master 2 accessible à http://www.picard.ups-tlse.fr/ toen/m2.html.

[To-Va] B. Toën, M. Vaquié, Moduli of objects in dg-categories, à paraître dans Ann. Scient. Ecole Norm. Sup., pré-publication arXiv math.AG/0503269.

[HAGI] B. Toën, G. Vezzosi, Homotopical algebraic geometry I: Topos theory, Adv. in Math. 193, (2005), 257-372.

[HAGII] B. Toën, G. Vezzosi, Homotopical algebraic geometry II: Geometric stacks and applications, à paraître dans Mémoires of the AMS, pré-publication math.AG/0404373. 